Supporting Information

\title{
Assembled Multinuclear Ruthenium(II)-NNNN Complexes: Synthesis, Catalytic Properties, and DFT Calculations
}

Tingting Liu, ${ }^{\dagger, \downarrow, \S}$ Kaikai Wu,${ }^{\dagger}$ Liandi Wang, ${ }^{\dagger}$ Hongjun Fan, $,{ }^{\dagger},, \|$ Yong-Gui Zhou, ${ }^{*}, \dagger$ and

$$
\text { Zhengkun } \mathrm{Yu}^{*, \dagger, \perp}
$$

'Dalian Institute of Chemical Physics, Chinese Academy of Sciences, 457 Zhongshan Road, Dalian, Liaoning 116023, PR China

University of Chinese Academy of Sciences, Beijing 100049, PR China

§Institute of Chemistry Henan Academy of Sciences, Zhengzhou 450002, PR China

"State Key Laboratory of Molecular Reaction Dynamics, Dalian Institute of Chemical Physics, Chinese Academy of Sciences, 457 Zhongshan Road, Dalian 116023, PR China

${ }^{\perp}$ State Key Laboratory of Organometallic Chemistry, Shanghai Institute of Organic Chemistry, Chinese Academy of Sciences, 354 Fenglin Road, Shanghai 200032, PR China

E-mail: zkyu@dicp.ac.cn; fanhj@dicp.ac.cn; ygzhou@dicp.ac.cn

\section{$\underline{\text { Experimental procedures and analytical data }}$}

Contents:

page

1. General considerations

2. Analytical data

3. Copies of NMR spectra

4. Copies of MALDI-TOF mass spectra

5. Computational details

6. References

\section{General considerations}


All the manipulations of air- and/or moisture-sensitive compounds were carried out under nitrogen atmosphere using the standard Schlenk techniques. The solvents were dried and distilled prior to use by the literature methods. ${ }^{1} \mathrm{H}$ and ${ }^{13} \mathrm{C}\left\{{ }^{1} \mathrm{H}\right\}$ NMR spectra were recorded on a Bruker DRX-400 spectrometer and all chemical shift values refer to $\delta_{\mathrm{TMS}}=0.00 \mathrm{ppm}, \mathrm{CDCl}_{3}\left(\delta\left({ }^{1} \mathrm{H}\right), 7.26 \mathrm{ppm} ; \delta\left({ }^{13} \mathrm{C}\right), 77.16 \mathrm{ppm}\right)$ and DMSO- $d_{6}\left(\delta\left({ }^{1} \mathrm{H}\right), 2.50 \mathrm{ppm} ; \delta\left({ }^{13} \mathrm{C}\right), 39.52 \mathrm{ppm}\right)$. Elemental analysis was achieved by the Analysis Center, Dalian University of Technology and Dalian Institute of Chemical Physics, Chinese Academy of Sciences. Matrix-Assisted Laser Desorption/ Ionization Time of Flight mass spectra were recorded on a MALDI-TOF/TOF 5800 spectrometer. All the melting points were uncorrected. TLC analysis was performed by using glass-backed plates coated with $0.2 \mathrm{~mm}$ silica gel. Flash column chromatography was performed on silica gel (200-300 meshes). All chemical reagents were purchased from commercial sources and used as received unless otherwise indicated. Ligands $\mathbf{2} \mathbf{a},{ }^{1} \mathbf{2} \mathbf{b},{ }^{2} \mathbf{5 a},{ }^{3}$ and $\mathbf{5} \mathbf{b}^{4}$ were prepared by the literature methods and their spectroscopic features are in good agreement with those reported in the literature.

\section{Analytical data}

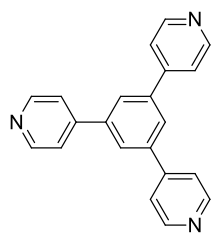

1,3,5-Tri(pyridin-4-yl)benzene (2a): ${ }^{1} \mathrm{H}$ NMR (400 MHz, $\left.\mathrm{CDCl}_{3}, 23{ }^{\circ} \mathrm{C}\right) \delta 8.76$ $(\mathrm{dd}, J=4.5,1.4 \mathrm{~Hz}, 6 \mathrm{H}), 7.92(\mathrm{~s}, 3 \mathrm{H}), 7.61(\mathrm{dd}, J=4.5,1.5 \mathrm{~Hz}, 6 \mathrm{H})$.

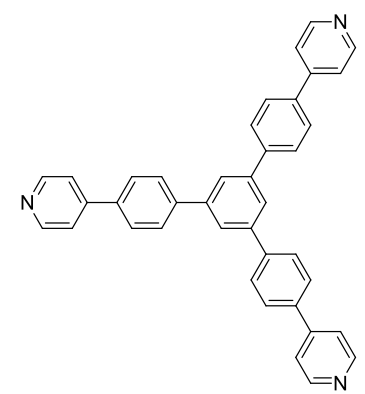

4,4'-(5'-(4-(Pyridin-4-yl)phenyl)-[1,1':3',1''-terphenyl]-4,4' '-diyl)dipyridine (2b): ${ }^{1} \mathrm{H}$ NMR $\left(400 \mathrm{MHz}, \mathrm{CDCl}_{3}, 23{ }^{\circ} \mathrm{C}\right) \delta 8.71(\mathrm{dd}, J=4.7,1.3 \mathrm{~Hz}, 6 \mathrm{H}), 7.91(\mathrm{~s}, 3$ H), $7.86(\mathrm{~d}, J=8.3 \mathrm{~Hz}, 6 \mathrm{H}), 7.80(\mathrm{~d}, J=8.3 \mathrm{~Hz}, 6 \mathrm{H}), 7.59(\mathrm{dd}, J=4.7,1.5 \mathrm{~Hz}, 6 \mathrm{H})$. 


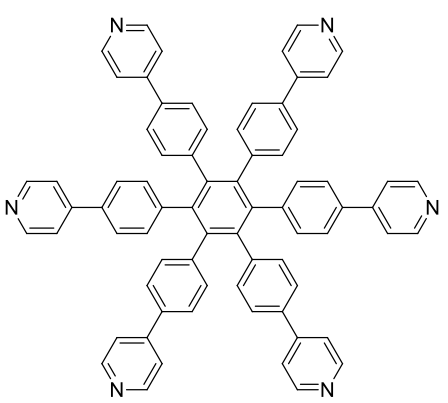

Ligand 5a: ${ }^{1} \mathrm{H} \mathrm{NMR}\left(400 \mathrm{MHz}, \mathrm{CDCl}_{3}, 23{ }^{\circ} \mathrm{C}\right) \delta 8.53(\mathrm{~d}, J=5.7 \mathrm{~Hz}, 1 \mathrm{H}), 7.32$ $(\mathrm{d}, J=5.8 \mathrm{~Hz}, 1 \mathrm{H}), 7.24(\mathrm{~s}, 1 \mathrm{H}), 7.02(\mathrm{~d}, J=8.2 \mathrm{~Hz}, 1 \mathrm{H})$.

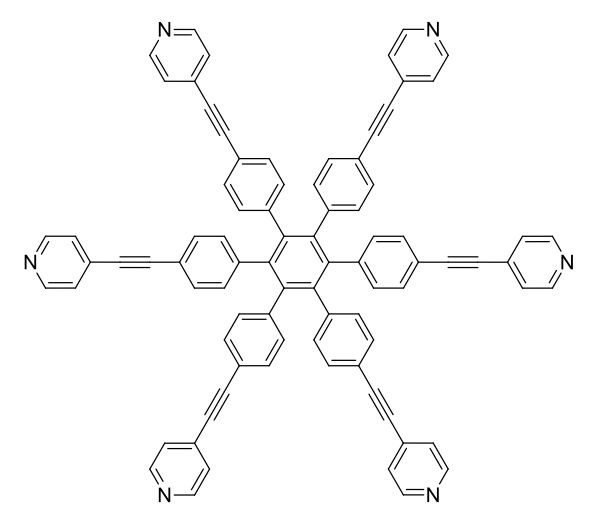

Ligand 5b: ${ }^{1} \mathrm{H}$ NMR $\left(400 \mathrm{MHz}, \mathrm{CDCl}_{3}, 23{ }^{\circ} \mathrm{C}\right) \delta 8.54(\mathrm{~s}, 6 \mathrm{H}), 7.28(\mathrm{~d}, J=4.8$ Hz, $6 \mathrm{H}), 7.15$ (d, $J=7.9 \mathrm{~Hz}, 6 \mathrm{H}), 6.87$ (d, $J=8.0 \mathrm{~Hz}, 6 \mathrm{H})$.

\section{Copies of NMR spectra}



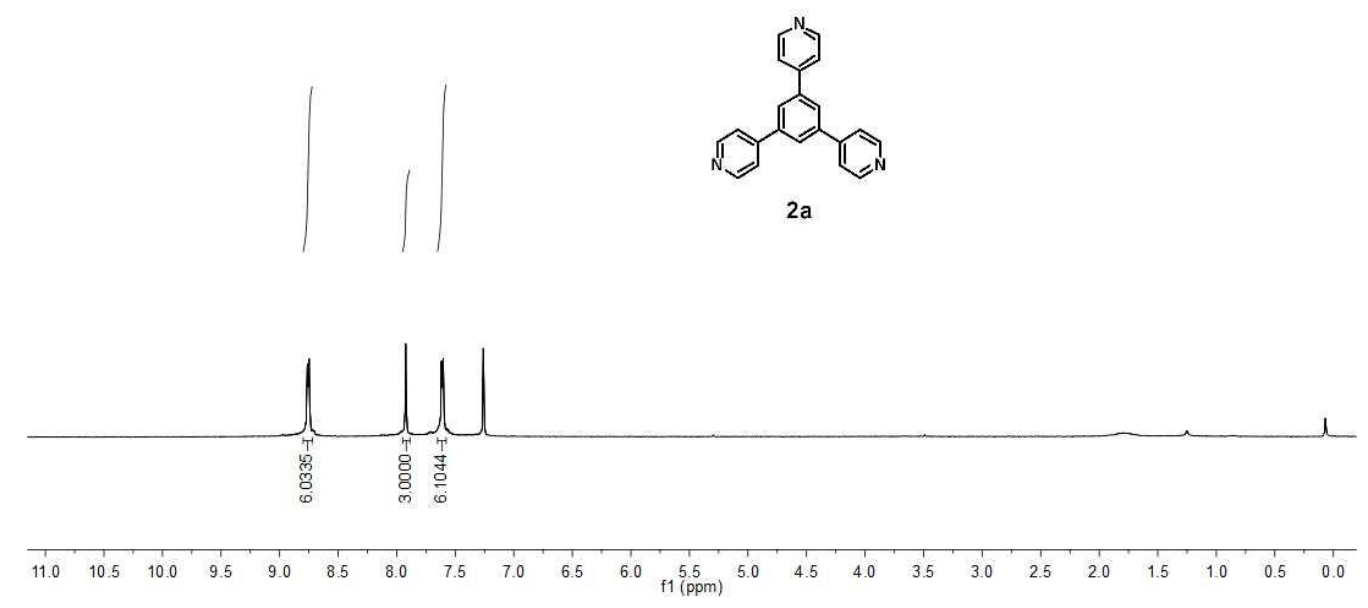

Figure S1. ${ }^{1} \mathrm{H}$ NMR spectrum of ligand $2 \mathbf{a}\left(400 \mathrm{MHz}, \mathrm{CDCl}_{3}, 23{ }^{\circ} \mathrm{C}\right)$.

1tt-482 IH NMR in CDC13

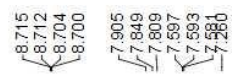
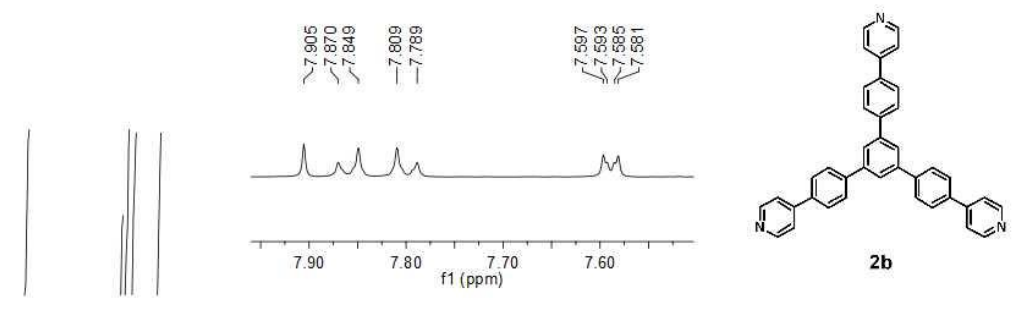

$\mathbf{2 b}$

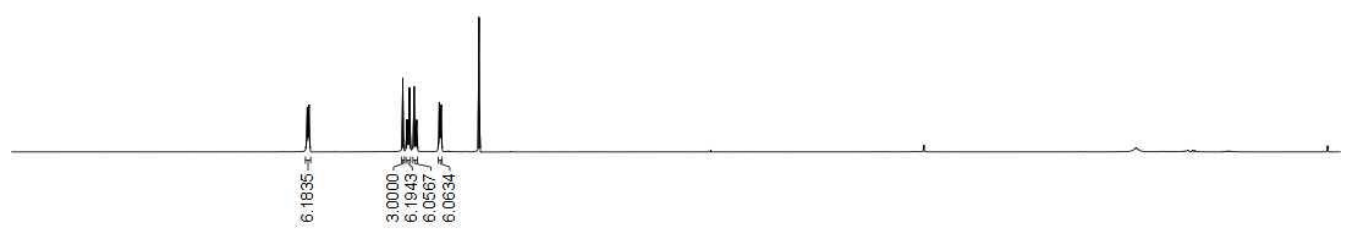

$\begin{array}{llllllllllllllllllllllllllllllllll}11.0 & 10.5 & 10.0 & 9.5 & 9.0 & 8.5 & 8.0 & 7.5 & 7.0 & 6.5 & 6.0 & 5.5 & 5.0 & 4.5 & 4.0 & 3.5 & 3.0 & 2.5 & 2.0 & 1.5 & 1.0 & 0.5 & 0.1\end{array}$

Figure S2. ${ }^{1} \mathrm{H}$ NMR spectrum of ligand $\mathbf{2 b}\left(400 \mathrm{MHz}, \mathrm{CDCl}_{3}, 23{ }^{\circ} \mathrm{C}\right)$. 


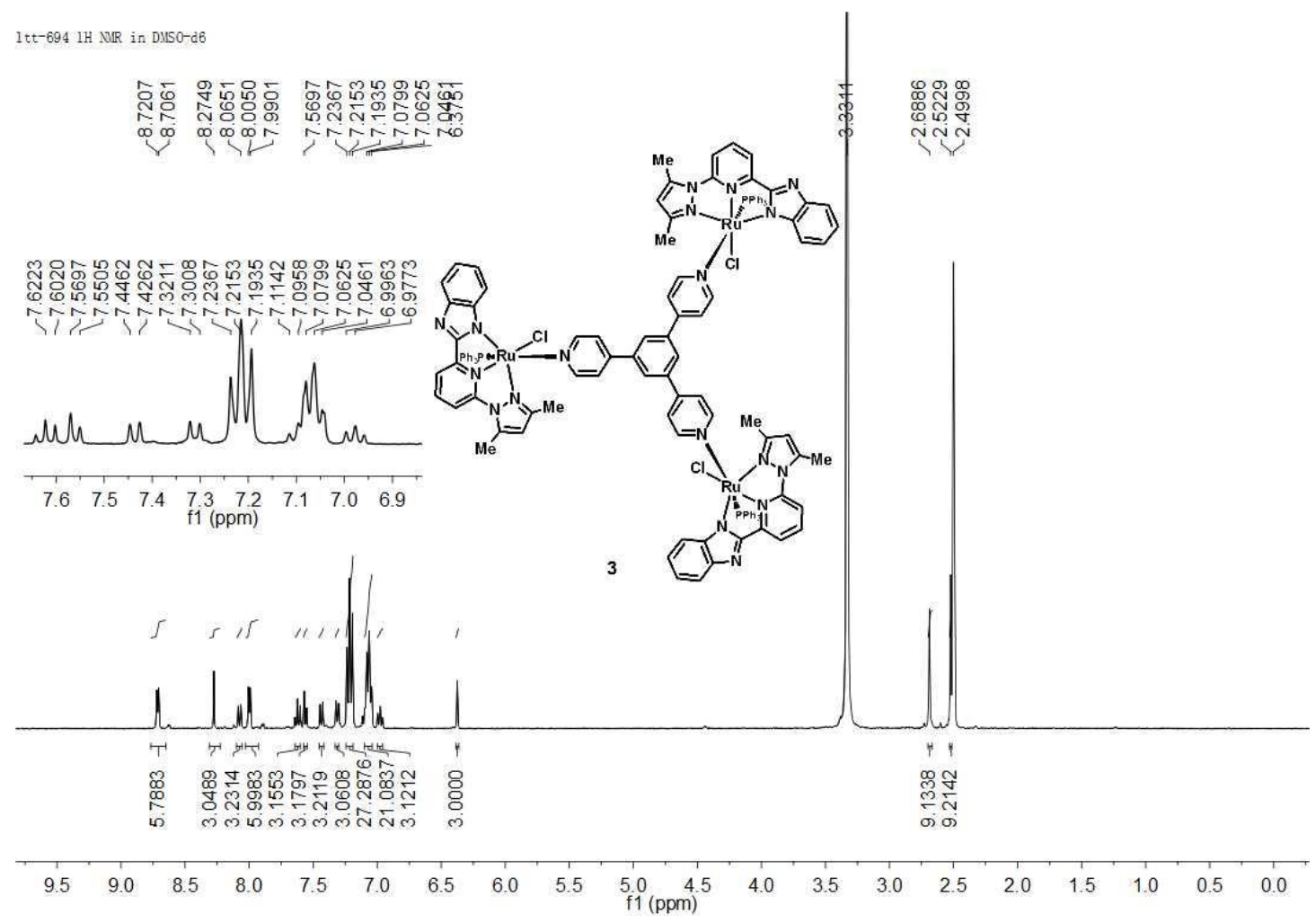

Figure S3. ${ }^{1} \mathrm{H}$ NMR spectrum of complex 3 (400 MHz, DMSO- $d_{6}, 23{ }^{\circ} \mathrm{C}$ ).

Itt-694 $13 \mathrm{C}$ NuR in Duso-d6

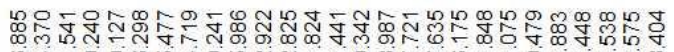

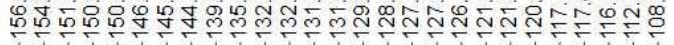

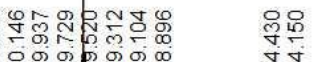

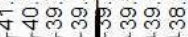

$\stackrel{i}{\frac{\pi}{2}}$

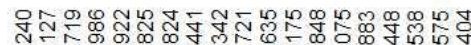

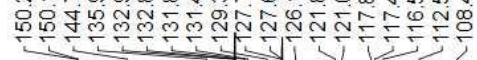
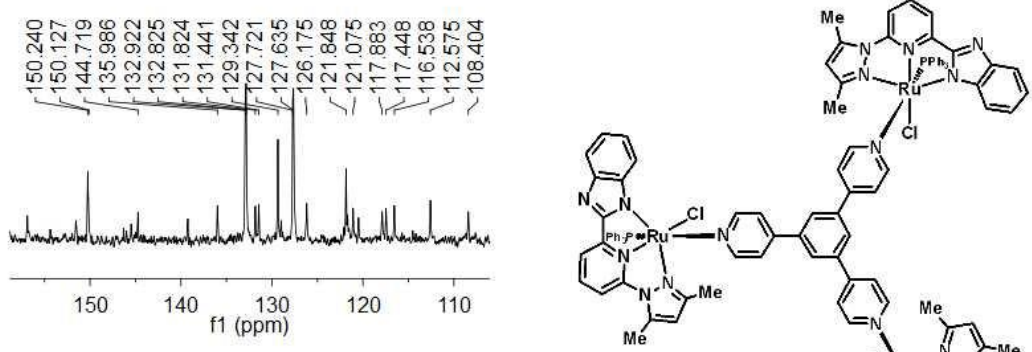

$\int^{n}$
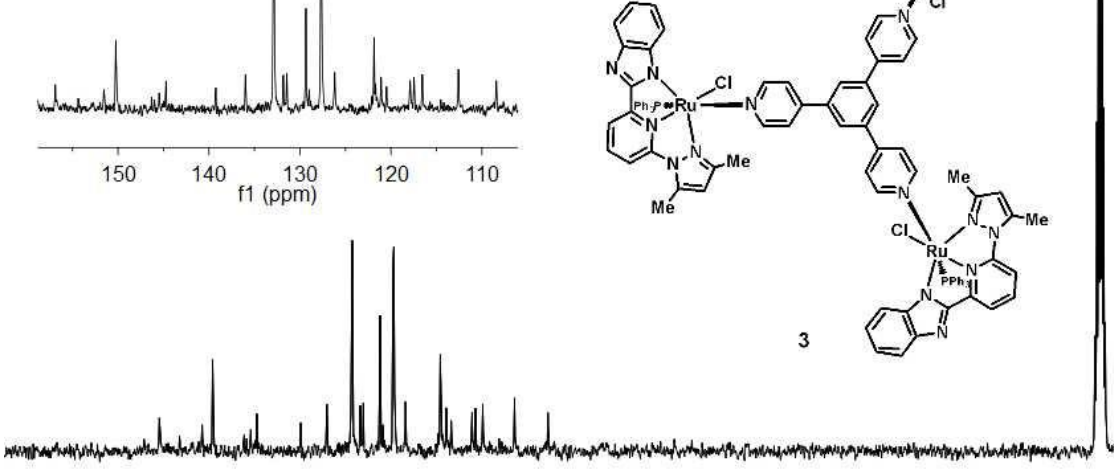

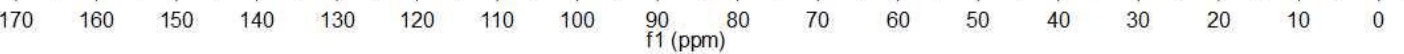

Figure S4. ${ }^{13} \mathrm{C}\left\{{ }^{1} \mathrm{H}\right\}$ NMR spectrum of complex $3\left(100 \mathrm{MHz}, \mathrm{DMSO}-d_{6}, 23{ }^{\circ} \mathrm{C}\right)$. 


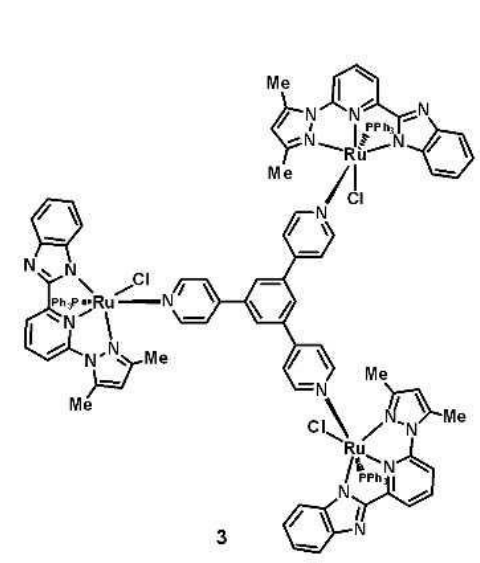

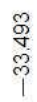

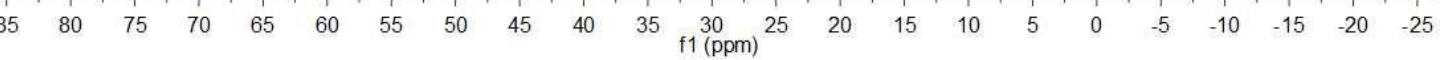

Figure S5. ${ }^{31} \mathrm{P}\left\{{ }^{1} \mathrm{H}\right\}$ NMR spectrum of complex $3\left(162 \mathrm{MHz}, \mathrm{DMSO}-d_{6}, 23{ }^{\circ} \mathrm{C}\right)$.

1 NWR $1 \mathrm{tt}-570$ in DUSO-d

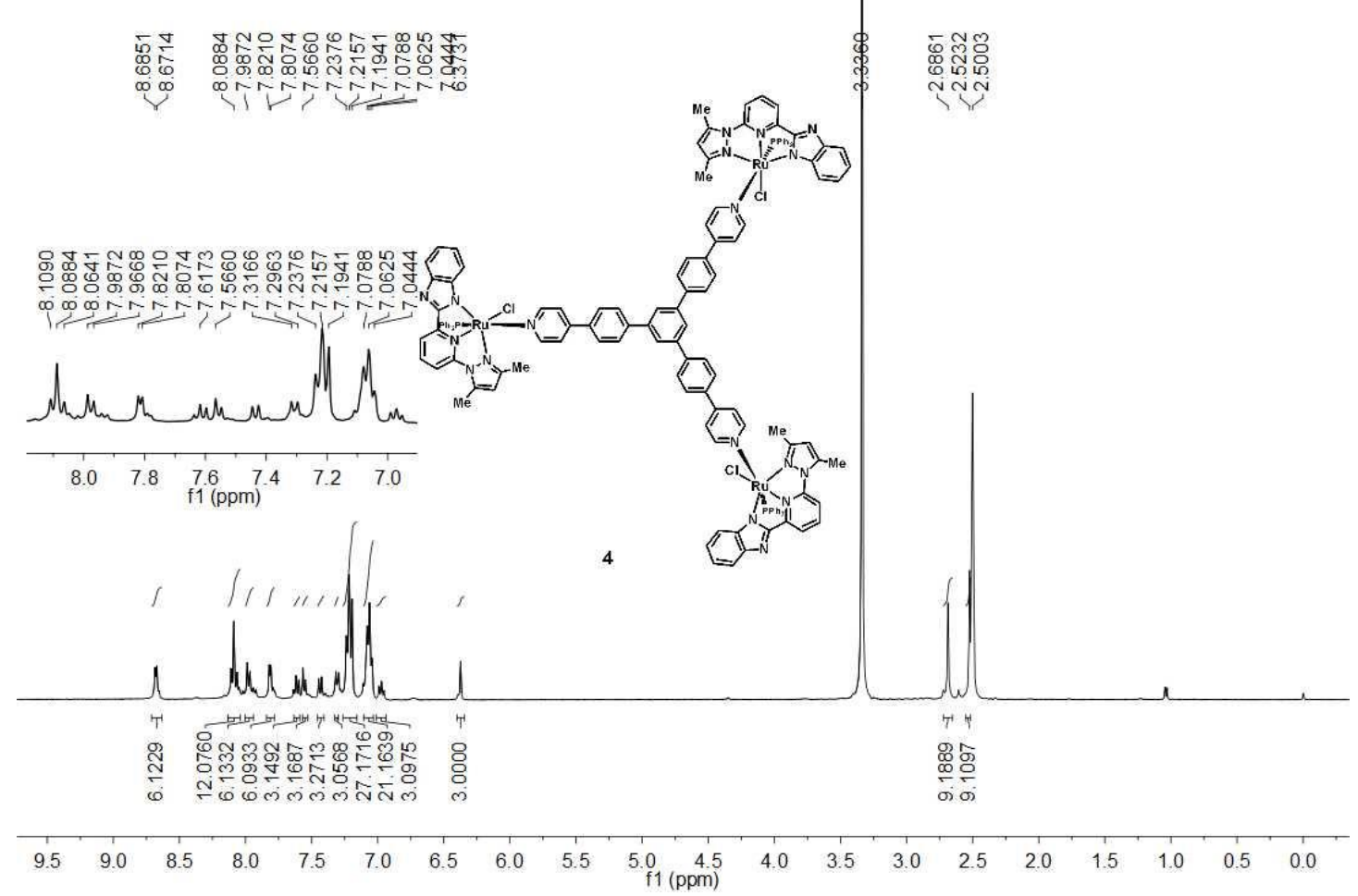

Figure S6. ${ }^{1} \mathrm{H}$ NMR spectrum of complex 4 (400 MHz, DMSO- $\left.d_{6}, 23{ }^{\circ} \mathrm{C}\right)$. 


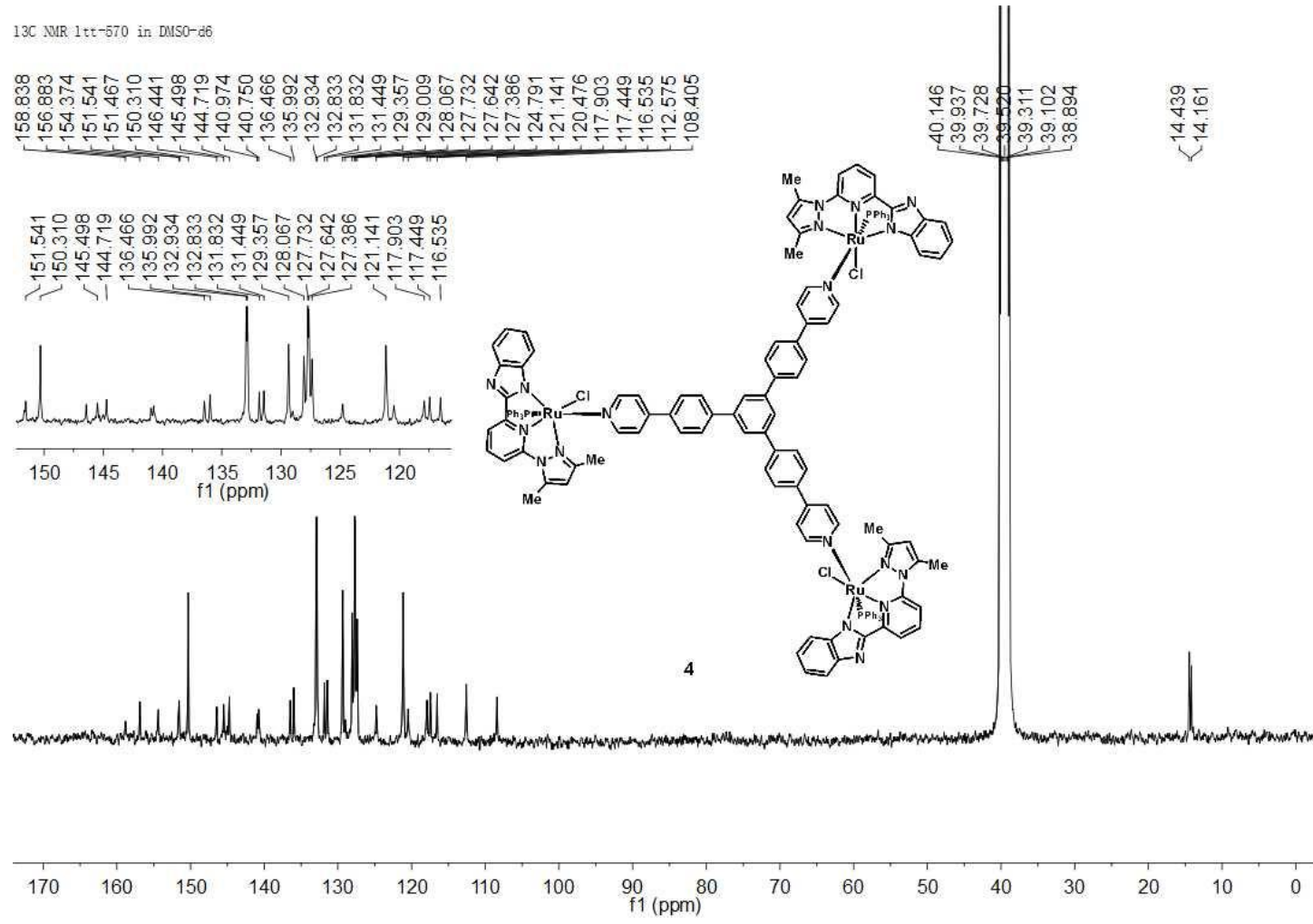

Figure S7. ${ }^{13} \mathrm{C}\left\{{ }^{1} \mathrm{H}\right\}$ NMR spectrum of complex $4\left(100 \mathrm{MHz}, \mathrm{DMSO}-d_{6}, 23{ }^{\circ} \mathrm{C}\right)$.

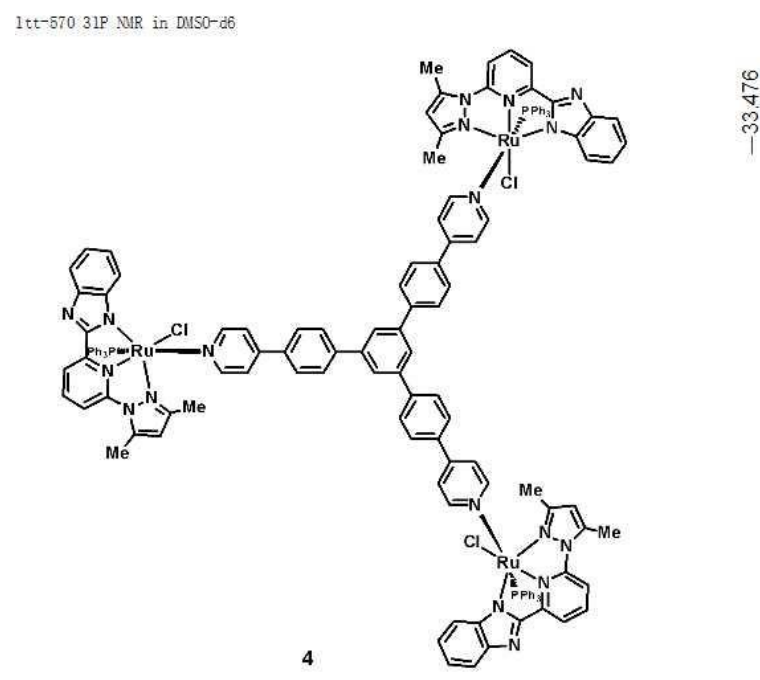

$\begin{array}{llllllllllllllllllllllllll}90 & 85 & 80 & 75 & 70 & 65 & 60 & 55 & 50 & 45 & 40 & 35 & 30 & 25 & 20 & 15 & 10 & 5 & 0 & -5 & -10 & -15 & -20\end{array}$

Figure S8. ${ }^{31} \mathrm{P}\left\{{ }^{1} \mathrm{H}\right\}$ NMR spectrum of complex $4\left(162 \mathrm{MHz}, \mathrm{DMSO}-d_{6}, 23{ }^{\circ} \mathrm{C}\right)$. 


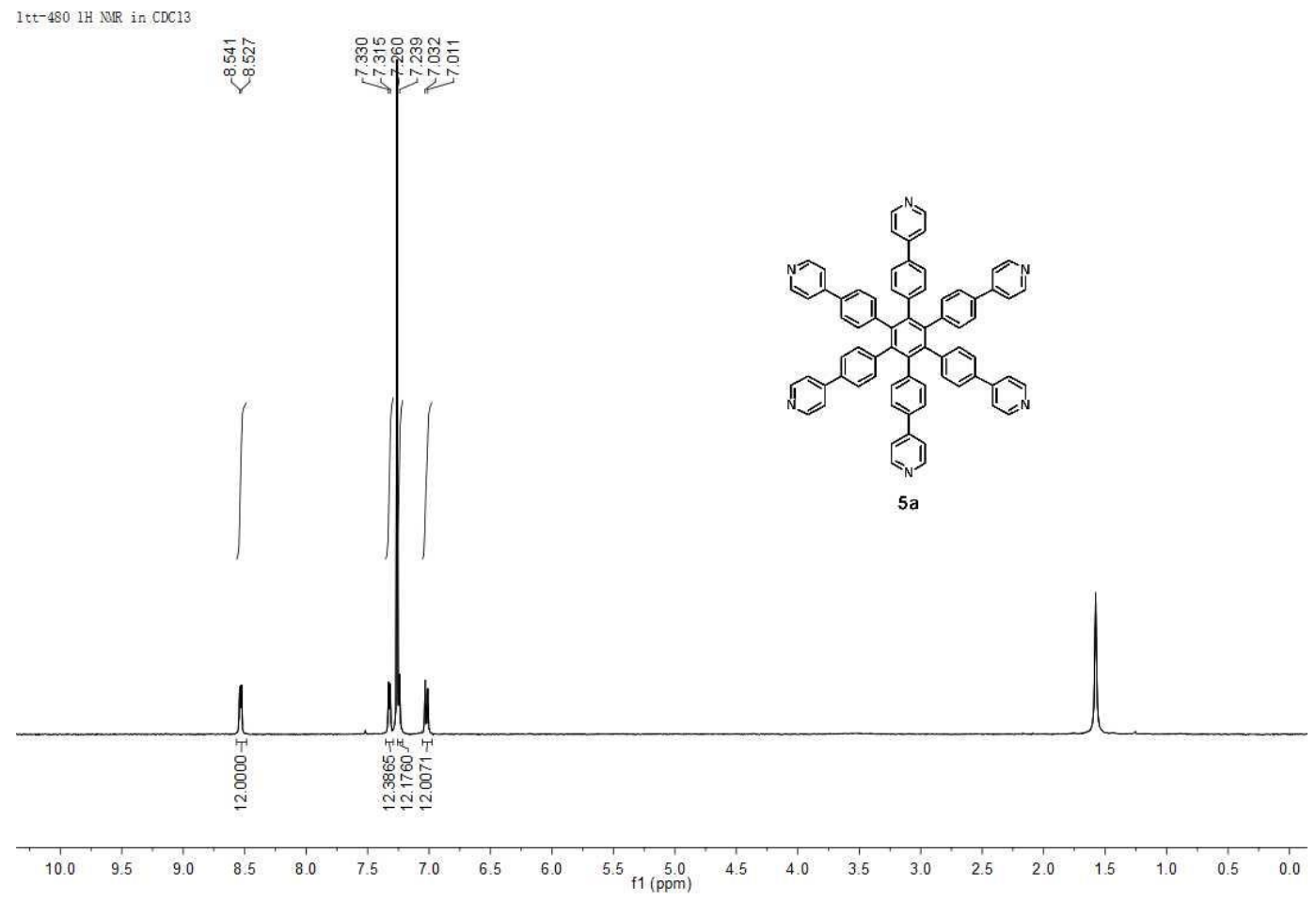

Figure S9. ${ }^{1} \mathrm{H}$ NMR spectrum of ligand $5 \mathbf{a}\left(400 \mathrm{MHz}, \mathrm{CDCl}_{3}, 23{ }^{\circ} \mathrm{C}\right)$.

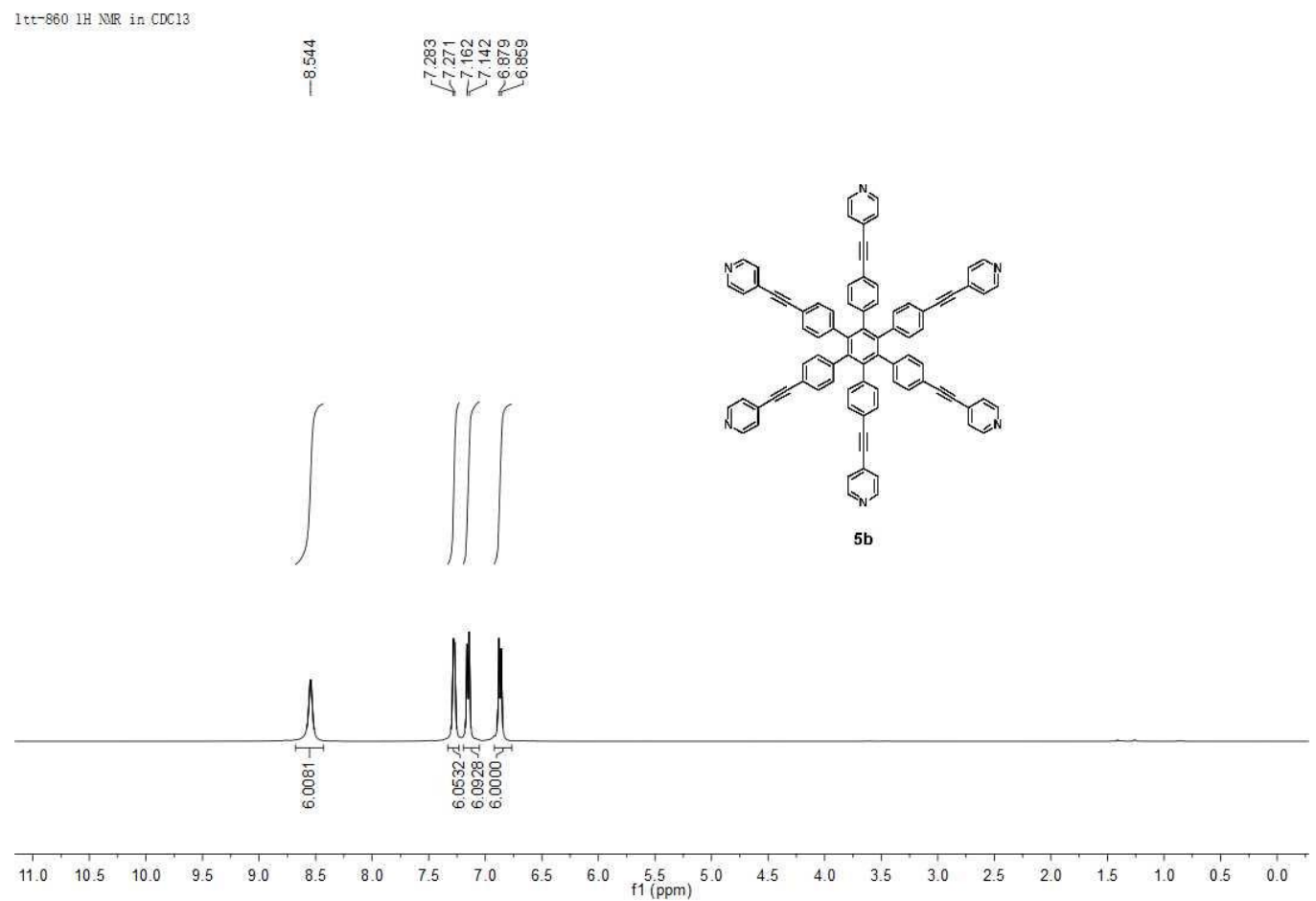

Figure S10. ${ }^{1} \mathrm{H}$ NMR spectrum of ligand $\mathbf{5 b}\left(400 \mathrm{MHz}, \mathrm{CDCl}_{3}, 23{ }^{\circ} \mathrm{C}\right)$. 


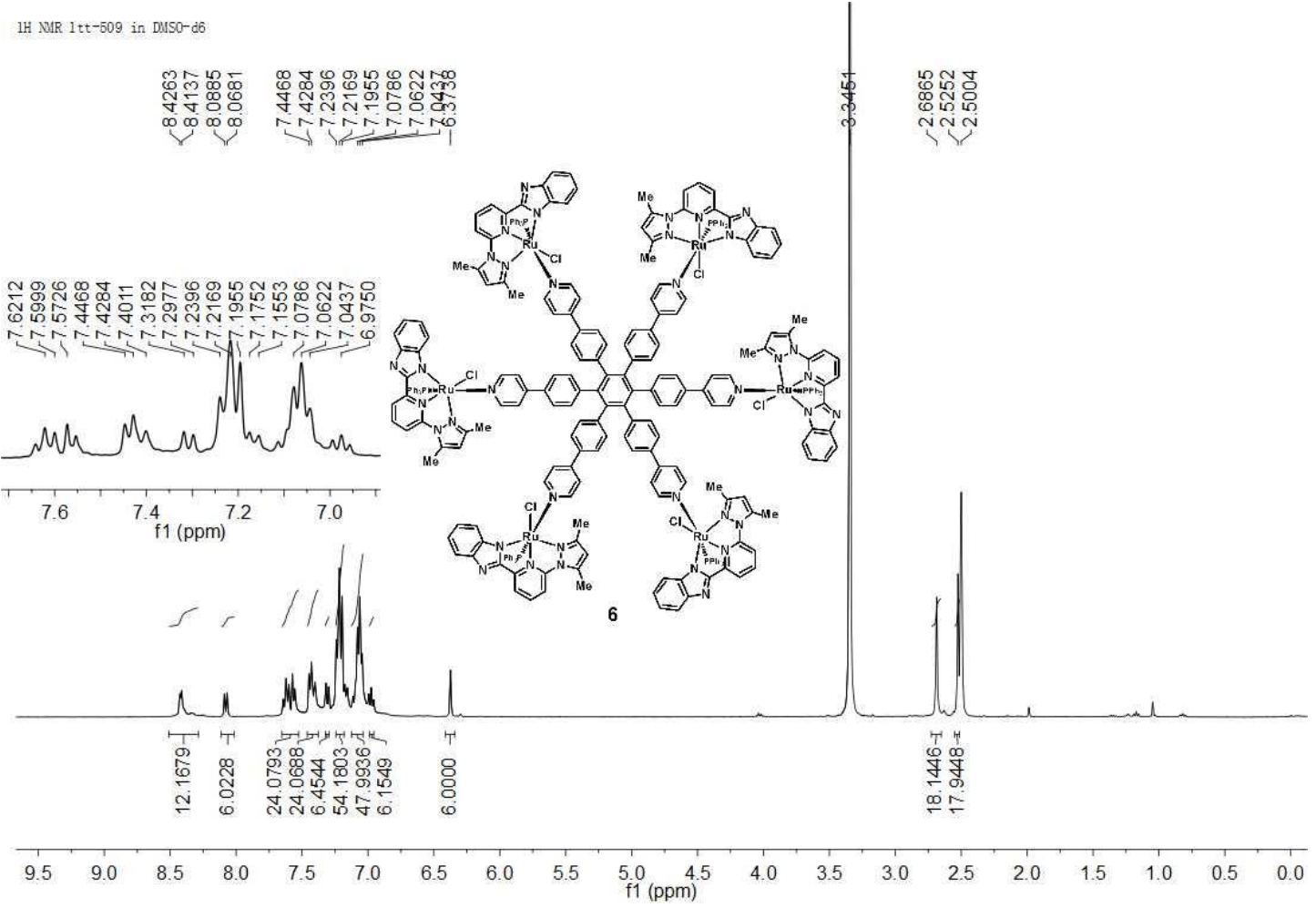

Figure S11. ${ }^{1} \mathrm{H}$ NMR spectrum of complex $6\left(400 \mathrm{MHz}, \mathrm{DMSO}-d_{6}, 23{ }^{\circ} \mathrm{C}\right)$.

$13 \mathrm{C}$ NMR $1 \mathrm{tt}-509$ in DUSO-d6

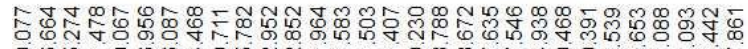

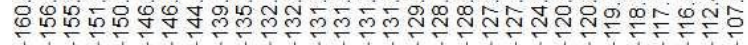

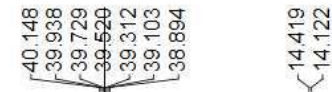
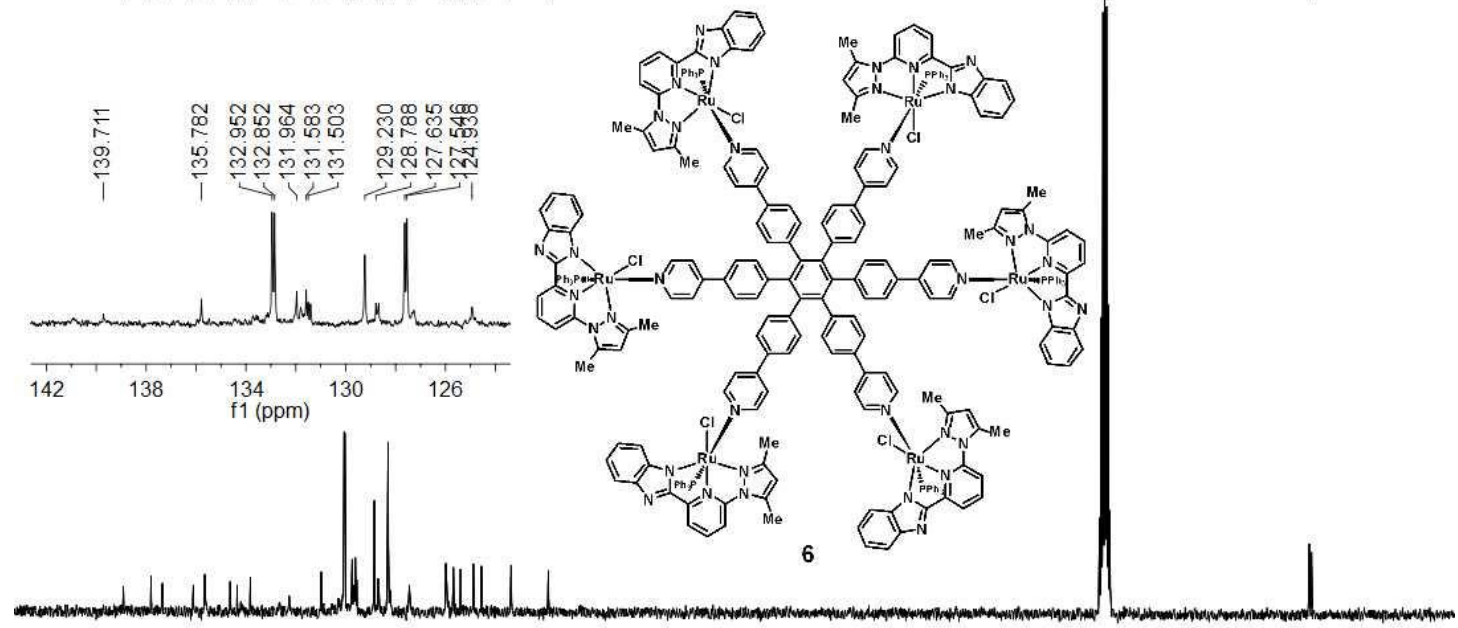

$\begin{array}{llllllllllllllllll}170 & 160 & 150 & 140 & 130 & 120 & 110 & 100 & \underset{\mathrm{f} 1}{9(\mathrm{ppm})} \mathbf{8 0} & 70 & 60 & 50 & 40 & 30 & 20 & 10 & 0\end{array}$

Figure S12. ${ }^{13} \mathrm{C}\left\{{ }^{1} \mathrm{H}\right\}$ NMR spectrum of complex $6\left(100 \mathrm{MHz}, \mathrm{DMSO}-d_{6}, 23{ }^{\circ} \mathrm{C}\right)$. 


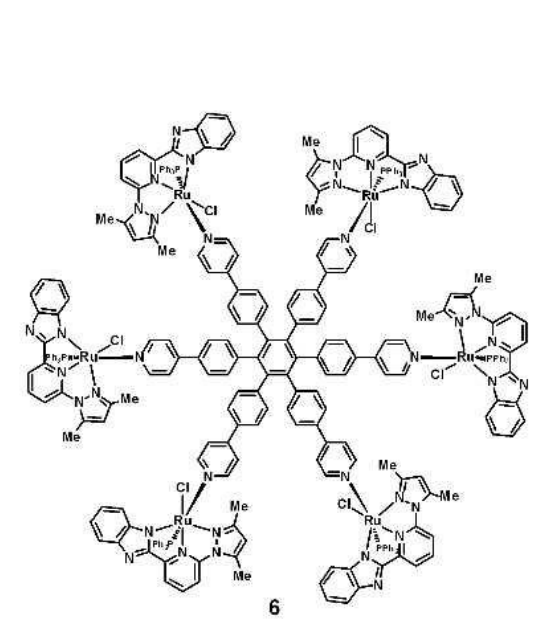

$\frac{6}{i n}$
0
0
1

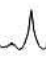

$\begin{array}{lllllllllllllllllllllllllllll}95 & 90 & 85 & 80 & 75 & 70 & 65 & 60 & 55 & 50 & 45 & 40 & \begin{array}{c}35 \\ \mathrm{f} 1(\mathrm{ppm})\end{array} & 30 & 25 & 20 & 15 & 10 & 5 & 0 & -5 & -10 & -15 & -20 & -25 & -31\end{array}$

Figure S13. ${ }^{31} \mathrm{P}\left\{{ }^{1} \mathrm{H}\right\}$ NMR spectrum of complex 6 (162 MHz, DMSO- $d_{6}, 23{ }^{\circ} \mathrm{C}$ ).

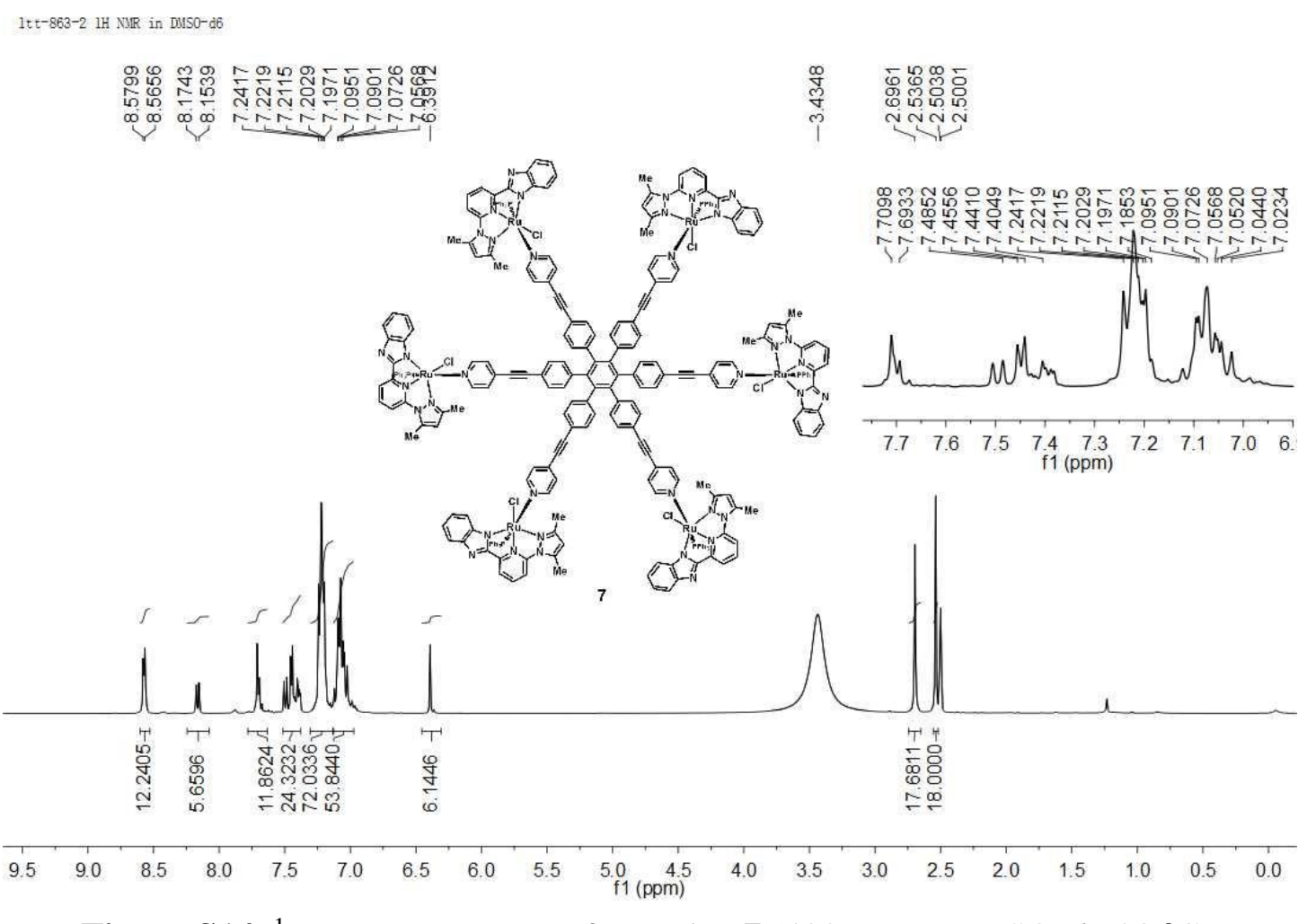

Figure S14. ${ }^{1} \mathrm{H}$ NMR spectrum of complex 7 (400 MHz, DMSO- $\left.d_{6}, 23{ }^{\circ} \mathrm{C}\right)$. 


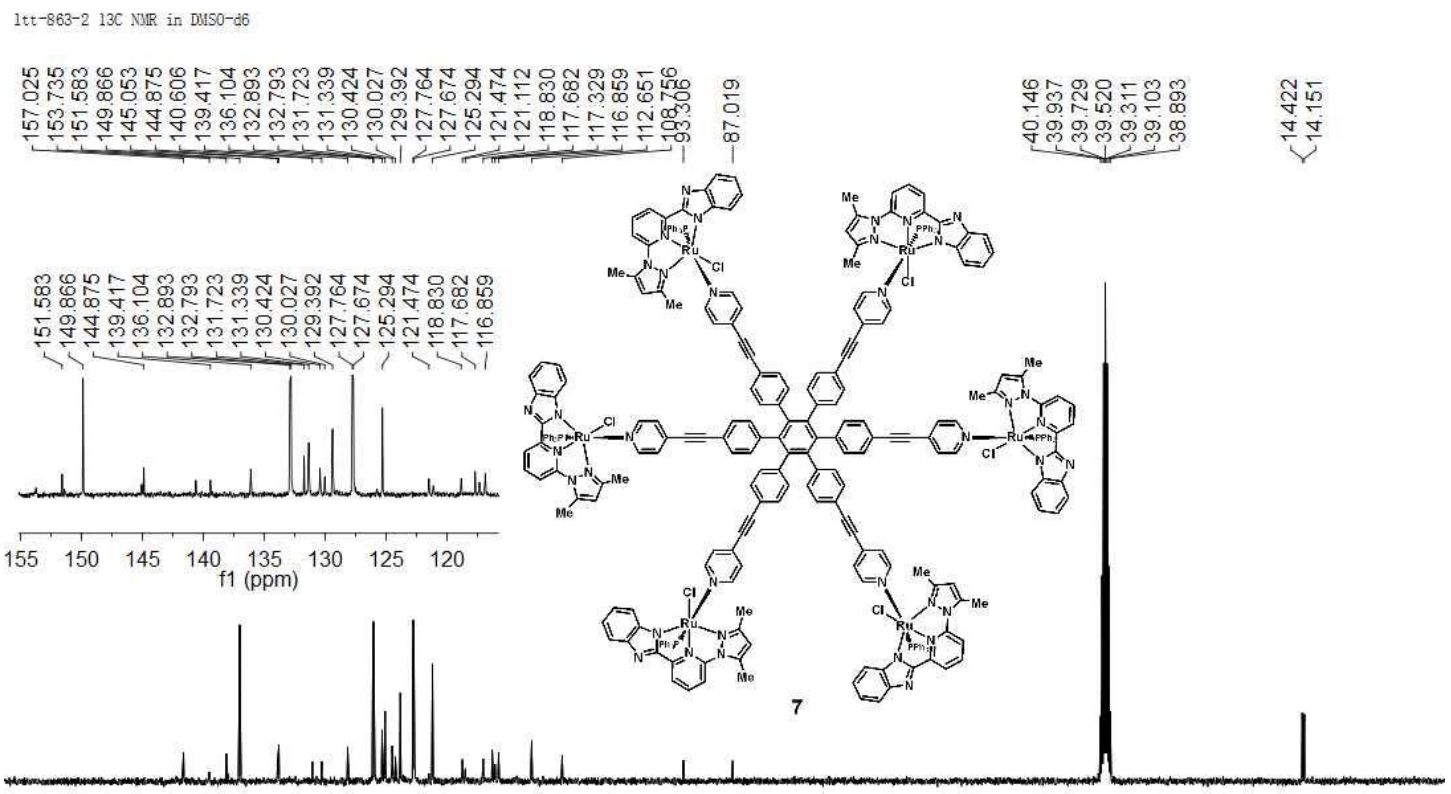

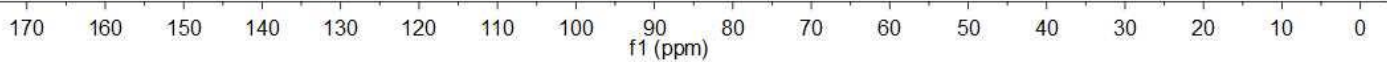

Figure S15. ${ }^{13} \mathrm{C}\left\{{ }^{1} \mathrm{H}\right\}$ NMR spectrum of complex 7 (100 MHz, DMSO- $\left.d_{6}, 23{ }^{\circ} \mathrm{C}\right)$.

LTT-863-2 31P NUR in DUSO-d6

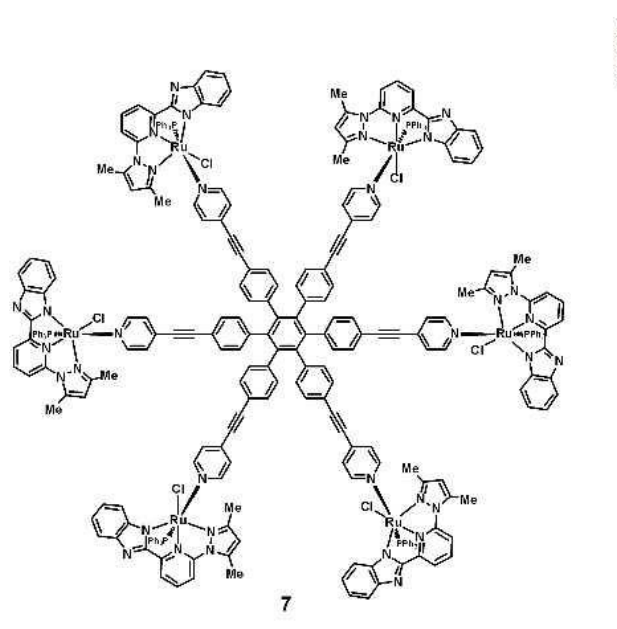

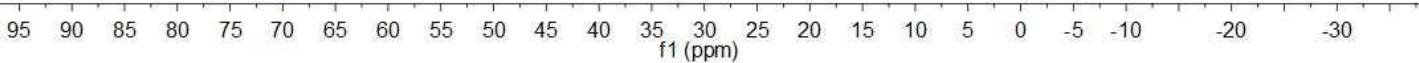

Figure S16. ${ }^{31} \mathrm{P}\left\{{ }^{1} \mathrm{H}\right\}$ NMR spectrum of complex 7 (162 MHz, DMSO- $\left.d_{6}, 23{ }^{\circ} \mathrm{C}\right)$. 


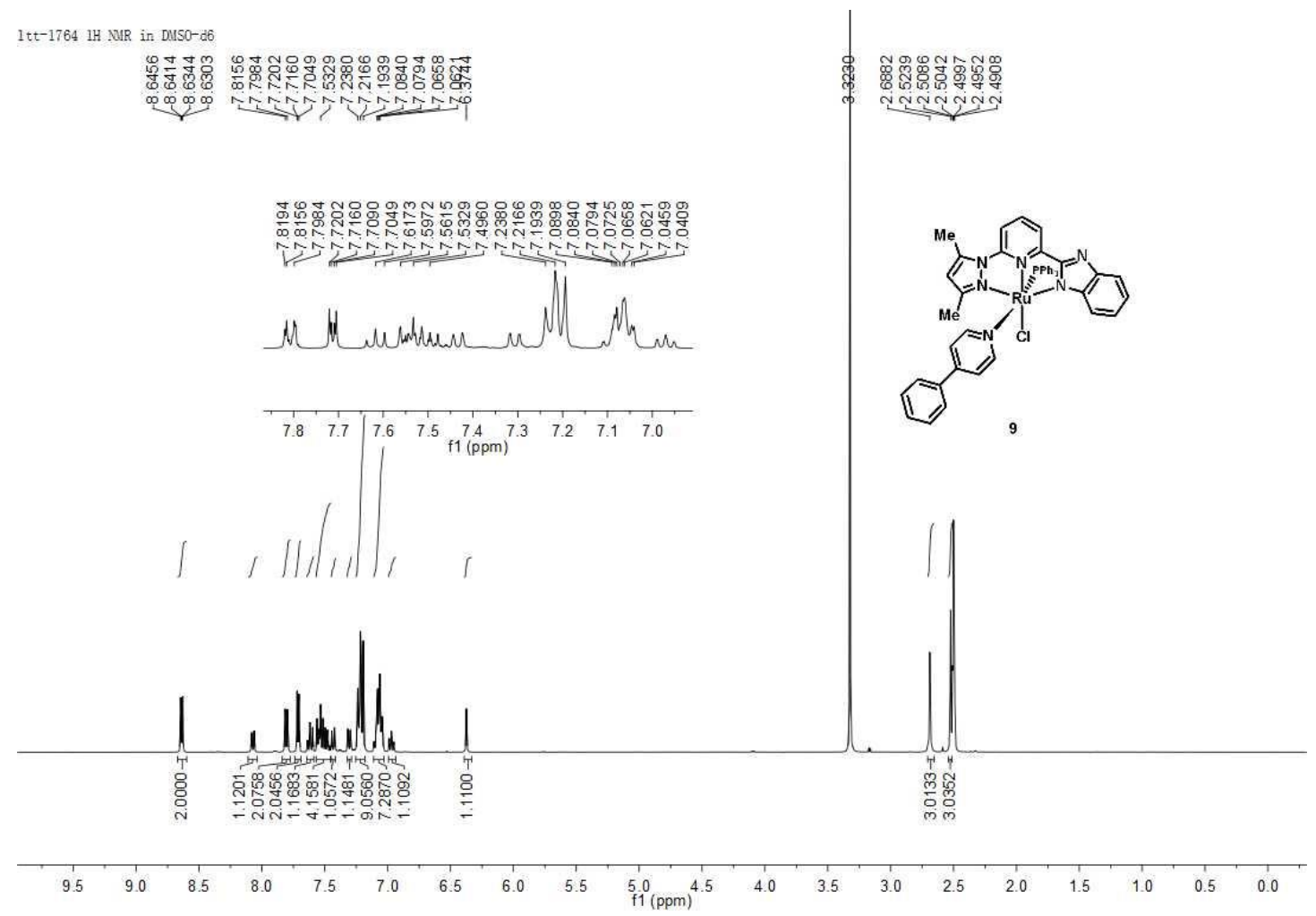

Figure S17. ${ }^{1} \mathrm{H}$ NMR spectrum of complex $9\left(400 \mathrm{MHz}, \mathrm{DMSO}-d_{6}, 23{ }^{\circ} \mathrm{C}\right)$.
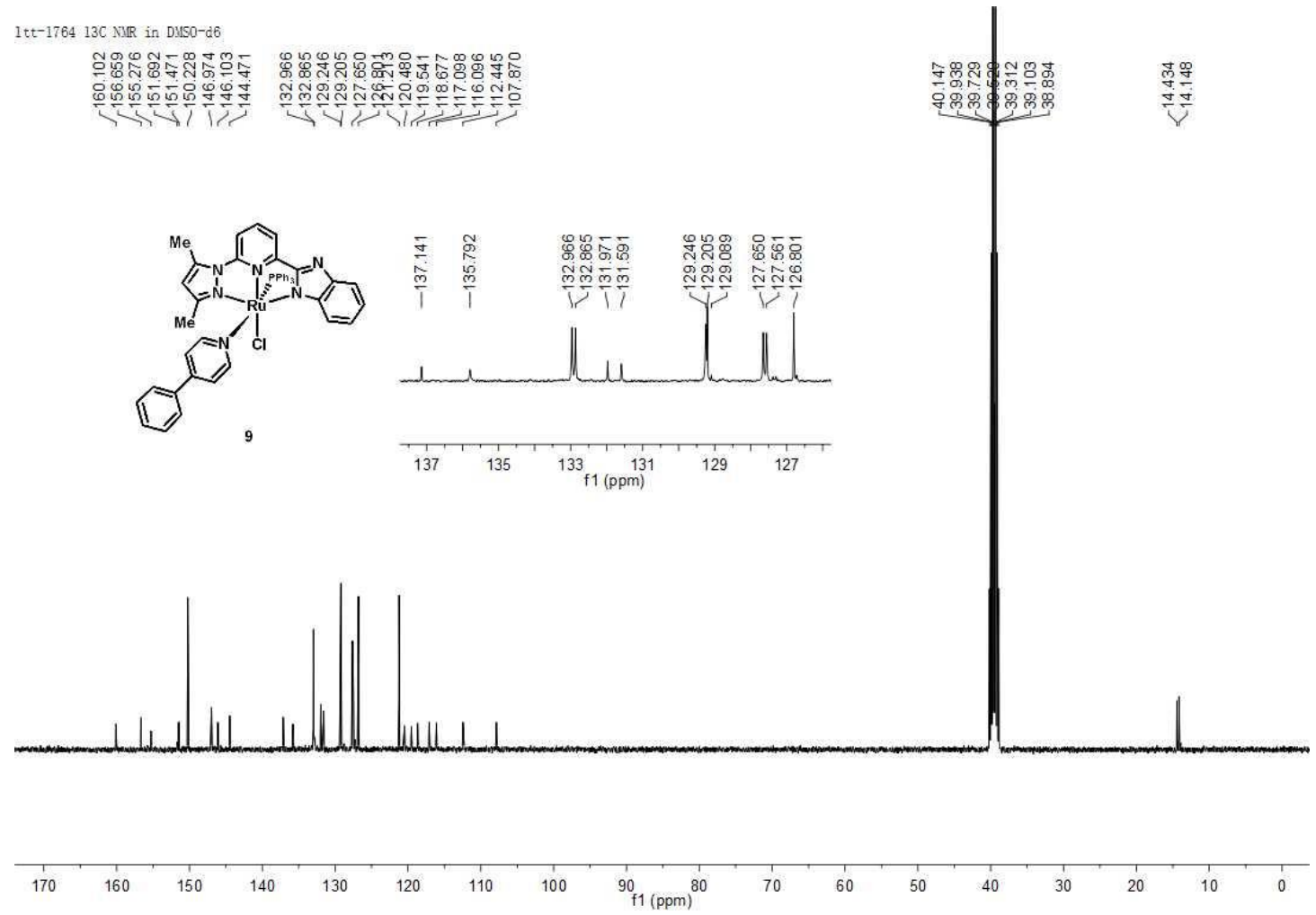

Figure S18. ${ }^{13} \mathrm{C}\left\{{ }^{1} \mathrm{H}\right\}$ NMR spectrum of complex 9 (100 MHz, DMSO- $d_{6}, 23{ }^{\circ} \mathrm{C}$ ). 

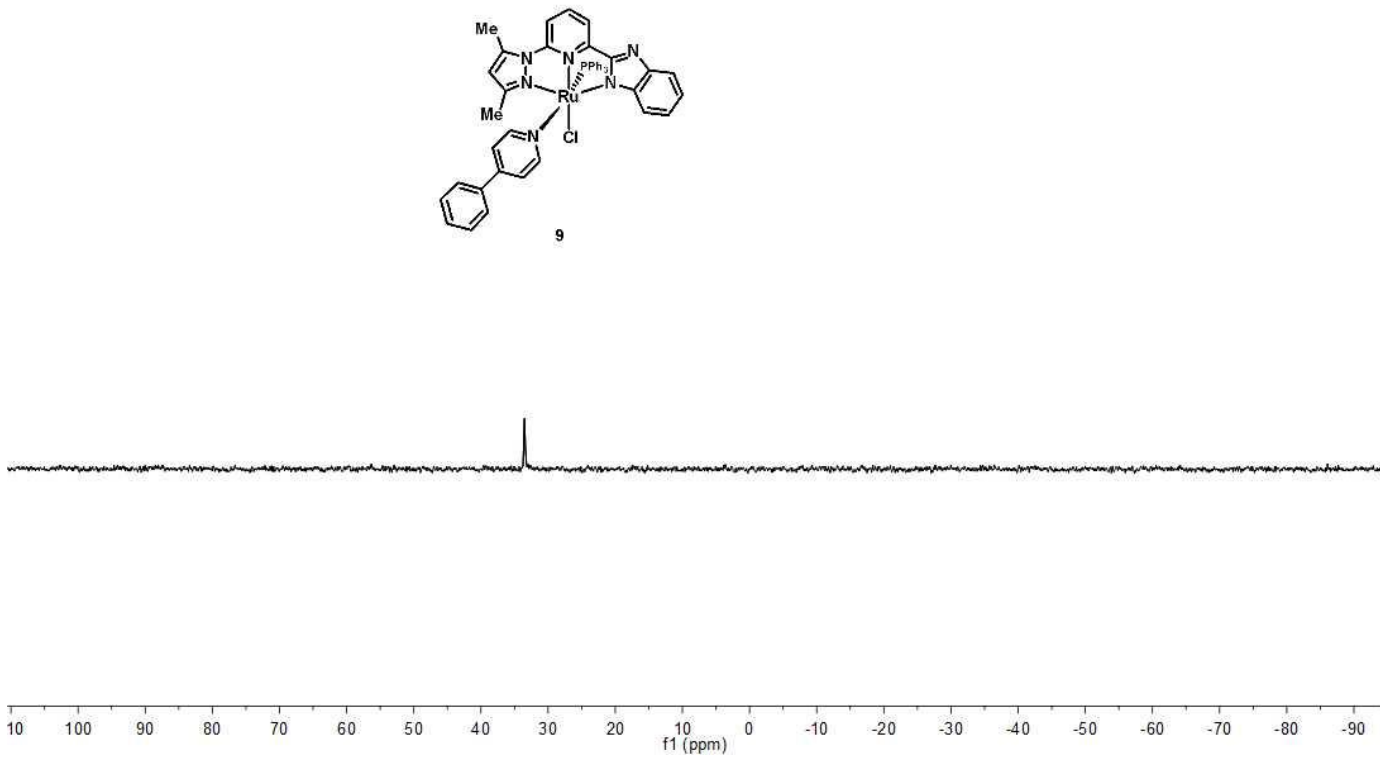

Figure S19. ${ }^{31} \mathrm{P}\left\{{ }^{1} \mathrm{H}\right\}$ NMR spectrum of complex 9 (162 MHz, DMSO- $d_{6}, 23{ }^{\circ} \mathrm{C}$ ).

\section{Copies of MALDI-TOF mass spectra}

The molecular ion peaks $[3+\mathrm{H}]^{+},[4+\mathrm{H}]^{+},[6+\mathrm{H}]^{+}$and $[7+\mathrm{H}]^{+}$were not observed in the mass spectra, while peaks corresponding to the fragments $[\mathbf{1 a}+\mathrm{H}]^{+},[\mathbf{2}+\mathrm{H}]^{+}$, and $[5+\mathrm{H}]^{+}$were observed, respectively. All the mass spectra of complexes $3, \mathbf{4}, \mathbf{6}$ and $\mathbf{7}$ revealed the protonated fragments of the mononuclear building block (1a) as well as the corresponding ligand (Figures S20-23). These fragment spectra were obtained by analyzing and fitting the mass spectra of $[3+\mathrm{H}]^{+},[4+\mathrm{H}]^{+},[6+\mathrm{H}]^{+}$, and $[7+\mathrm{H}]^{+}$by means of the Data Explore (TM) Software (Figure S24).

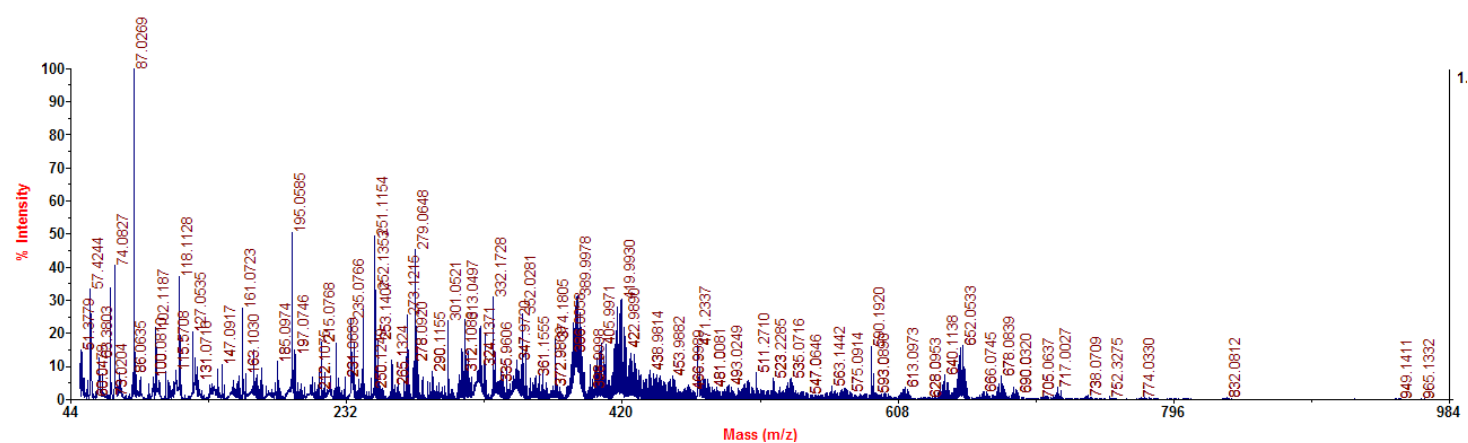

Figure S20. MALDI-TOF MS (m/z) spectrum of complex 3. 


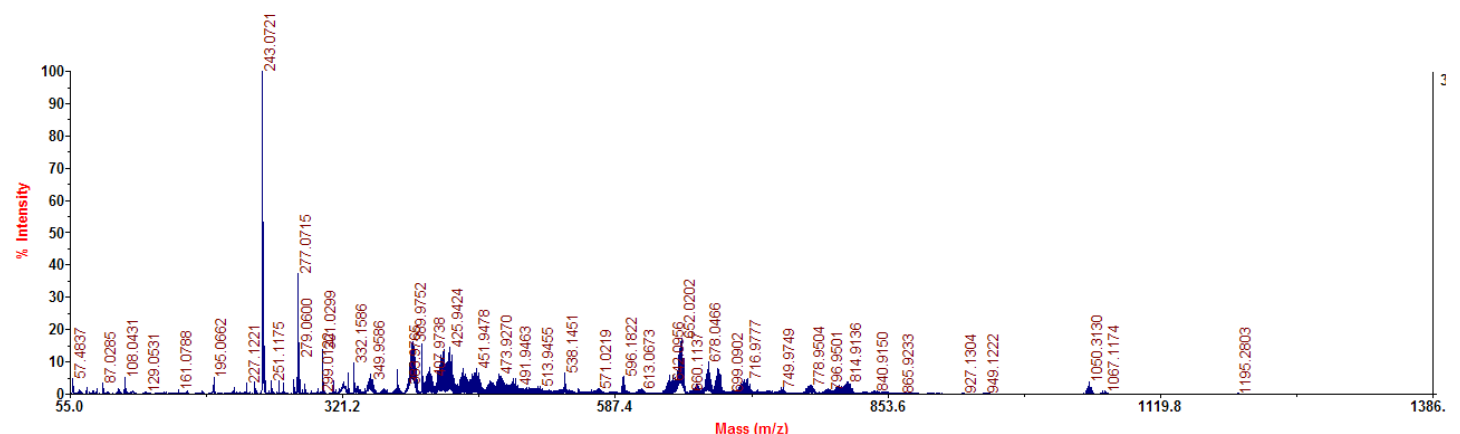

Figure S21. MALDI-TOF MS (m/z) spectrum of complex 4.

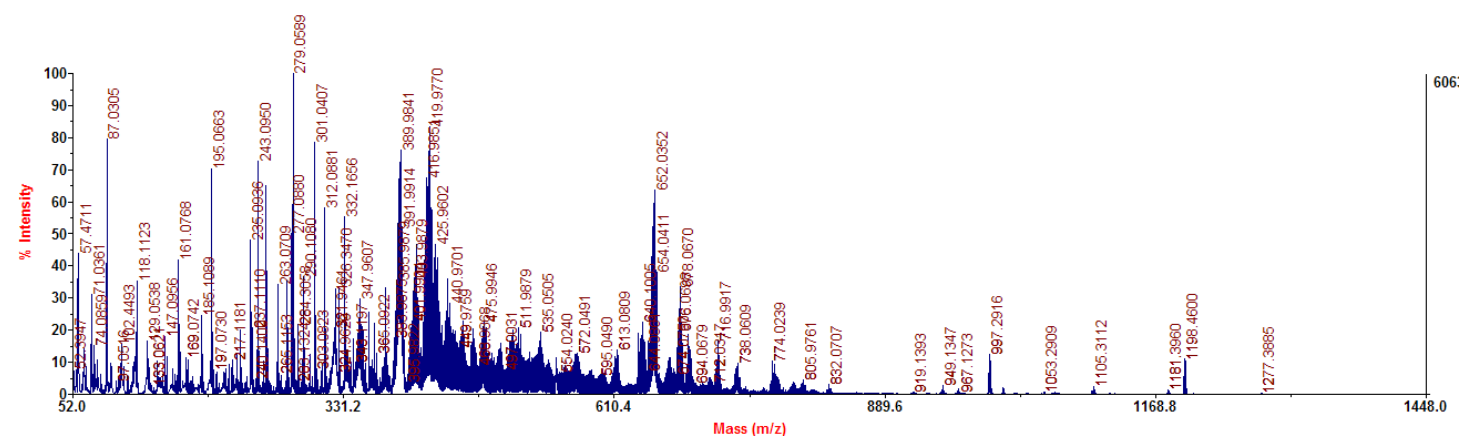

Figure S22. MALDI-TOF MS (m/z) spectrum of complex 6.

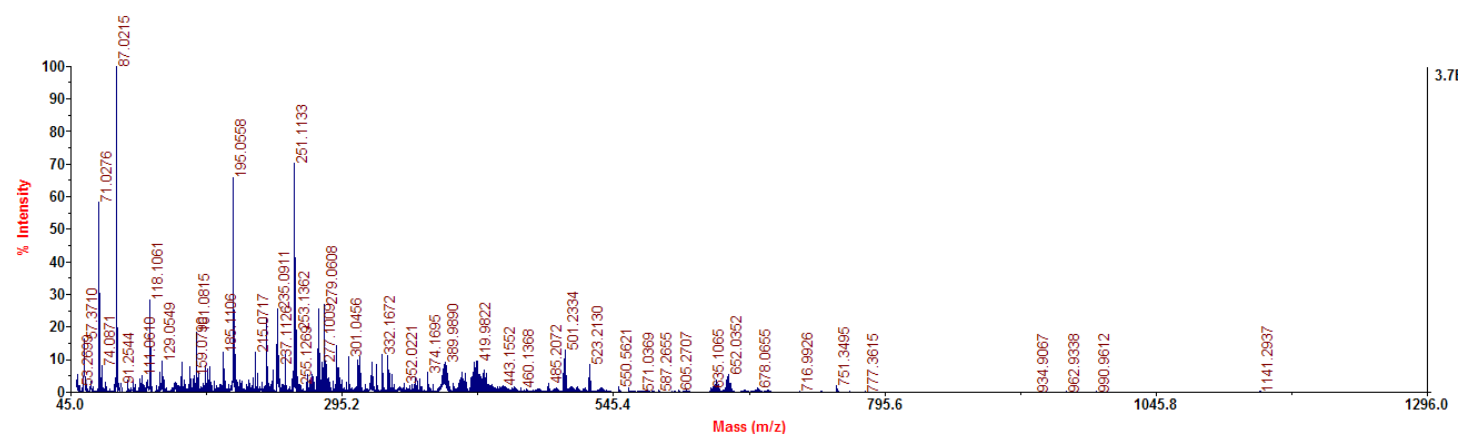

Figure S23. MALDI-TOF MS (m/z) spectrum of complex 7. 


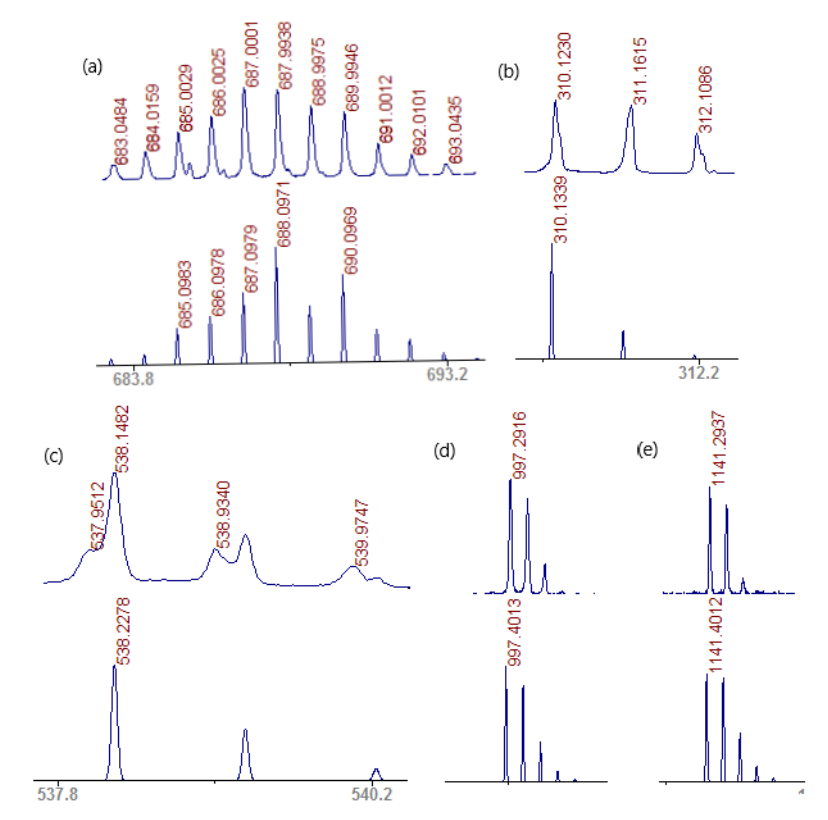

Figure S24. The MALDI-TOF mass spectra of the fragments from complexes 3, 4, 6, and 7. Fragments ( $\mathrm{m} / \mathrm{z}$ ): top - the experimental result, bottom - the theoretical stimulation. (a) $[\mathbf{1 a}+\mathrm{H}]^{+}$from complexes $\mathbf{3}, \mathbf{4}, \mathbf{6}$, and 7. (b) $[2 \mathbf{a}+\mathrm{H}]^{+}$from complex 3. (c) $[\mathbf{2 b}+\mathrm{H}]^{+}$from complex 4. (d) $[\mathbf{5 a}+\mathrm{H}]^{+}$from complex $\mathbf{6}$. (e) $[\mathbf{5 b}+\mathrm{H}]^{+}$from complex $\mathbf{7}$.

\section{Computational details}

The geometry optimizations and energy calculations were carried out by Density Functional Theory as implemented in the Gaussian $16^{5} a b$ initio quantum chemistry programs and M06-2 $\mathrm{x}^{6}$ functional. Ruthenium was represented using the def2-TZVP basis sets, and for all others atoms the cc- $\mathrm{Pvdz}^{8}$ basis set were used. No symmetry restrictions were applied during the geometry optimization.

The interaction energy between different groups were calculated by the M06-2 $\mathrm{x}^{6}$ functional and IQA method. ${ }^{9,10}$ These calculations were carried out by the Amsterdam Density Functional (ADF 2018) package, ${ }^{11}$ utilizing all-electron triple-basis set. Optimized geometries from Gaussian 16 calculations were adopted.

\section{Optimized Geometries}

$\begin{array}{ccccccc}\mathrm{Ru}(\mathrm{II})(\mathrm{Cl})\left(\mathrm{PPh}_{3}\right)-\mathrm{NNNN}\left(\mathrm{PPh}_{3} \text { trans to } 2 \mathrm{a}\right) & \mathrm{C} & -0.062766-1.753231 & 2.069222 \\ \mathrm{C} & -0.101513 & 0.564493 & 2.432843 & \mathrm{C} & -0.071535-2.010056 & 3.439684 \\ \mathrm{~N} & -0.063282 & -0.500661 & 1.600800 & \mathrm{C} & -0.115234-0.912431 & 4.305449\end{array}$




\begin{tabular}{|c|c|c|c|}
\hline $\mathrm{C}$ & $-0.139031 \quad 0.385847 \quad 3.816422$ & $\mathrm{C}$ & $7.171400-1.403891-0.689661$ \\
\hline $\mathrm{Ru}$ & $-0.022036-0.151061-0.379418$ & $\mathrm{C}$ & $8.563617-1.418584-0.703957$ \\
\hline $\mathrm{N}$ & $-0.065564-2.729662 \quad 1.043475$ & $\mathrm{C}$ & $9.279015-0.255770-0.420726$ \\
\hline $\mathrm{H}$ & $-0.046150-3.016235 \quad 3.838715$ & $\mathrm{C}$ & $8.594293 \quad 0.921335-0.120884$ \\
\hline $\mathrm{H}$ & $-0.131061-1.091877 \quad 5.380771$ & $\mathrm{C}$ & $7.202179 \quad 0.935473-0.100547$ \\
\hline $\mathrm{H}$ & $-0.172331 \quad 1.266697 \quad 4.455122$ & $\mathrm{H}$ & $6.616270-2.321426-0.891961$ \\
\hline $\mathrm{N}$ & $0.035112-2.309014-0.262667$ & $\mathrm{H}$ & $9.092597-2.344323-0.932955$ \\
\hline $\mathrm{C}$ & $0.088099-3.400476-1.009344$ & $\mathrm{H}$ & $10.369361-0.266474-0.434917$ \\
\hline $\mathrm{C}$ & $-0.004824-4.551765-0.186907$ & $\mathrm{H}$ & $\begin{array}{lll}9.146952 & 1.836989 & 0.091778\end{array}$ \\
\hline $\mathrm{C}$ & $-0.102330-4.103282 \quad 1.106531$ & $\mathrm{H}$ & $\begin{array}{lll}6.671161 & 1.865121 & 0.110399\end{array}$ \\
\hline $\mathrm{C}$ & $\begin{array}{lll}-0.036706 & 1.844167 & 1.737749\end{array}$ & $\mathrm{P}$ & $-2.332400-0.039138-0.454794$ \\
\hline $\mathrm{N}$ & $\begin{array}{lll}0.062051 & 1.819039 & 0.372228\end{array}$ & $\mathrm{C}$ & $-3.149792 \quad 1.376212-1.301863$ \\
\hline $\mathrm{C}$ & $\begin{array}{lll}0.284078 & 3.125858 & 0.048014\end{array}$ & $\mathrm{C}$ & $\begin{array}{lll}-3.034926 & 0.104449 & 1.247199\end{array}$ \\
\hline $\mathrm{C}$ & $\begin{array}{lll}0.259892 & 3.872961 & 1.264920\end{array}$ & $\mathrm{C}$ & $-3.224330-1.467007-1.214115$ \\
\hline $\mathrm{N}$ & $\begin{array}{lll}0.063731 & 3.031836 & 2.327683\end{array}$ & $\mathrm{C}$ & $-4.545300 \quad 1.507897-1.258530$ \\
\hline $\mathrm{C}$ & $\begin{array}{lll}0.456201 & 5.269175 & 1.239832\end{array}$ & $\mathrm{C}$ & $-5.161901 \quad 2.598058-1.862249$ \\
\hline $\mathrm{C}$ & $\begin{array}{lll}0.692524 & 5.869794 & 0.018783\end{array}$ & $\mathrm{C}$ & $-4.390210 \quad 3.566233-2.511308$ \\
\hline $\mathrm{C}$ & $0.753398 \quad 5.112612-1.181055$ & $\mathrm{C}$ & $-3.006385 \quad 3.432866-2.561896$ \\
\hline $\mathrm{C}$ & $0.557864 \quad 3.744592-1.189073$ & $\mathrm{C}$ & $-2.384582 \quad 2.334850-1.963112$ \\
\hline $\mathrm{C}$ & $0.260012-3.348047-2.490510$ & $\mathrm{C}$ & $-4.404306-2.026046-0.709353$ \\
\hline $\mathrm{H}$ & $-0.006227-5.588503-0.507482$ & $\mathrm{C}$ & $-5.042911-3.059298-1.395721$ \\
\hline $\mathrm{C}$ & $-0.237565-4.918857 \quad 2.349096$ & $\mathrm{C}$ & $-4.518703-3.532471-2.596998$ \\
\hline $\mathrm{H}$ & $\begin{array}{lll}0.429449 & 5.841439 & 2.167840\end{array}$ & $\mathrm{C}$ & $-3.356315-2.962414-3.115967$ \\
\hline $\mathrm{H}$ & $0.851505 \quad 6.948062-0.029990$ & $\mathrm{C}$ & $-2.708087-1.937603-2.428769$ \\
\hline $\mathrm{H}$ & $0.972736 \quad 5.626948-2.118057$ & $\mathrm{C}$ & $-3.254587 \quad 1.374002 \quad 1.796340$ \\
\hline $\mathrm{H}$ & $0.634019 \quad 3.148518-2.100968$ & $\mathrm{C}$ & $\begin{array}{lll}-3.620169 & 1.510902 & 3.135395\end{array}$ \\
\hline $\mathrm{H}$ & $-0.560103-3.886370-2.985930$ & $\mathrm{C}$ & $-3.772479 \quad 0.383930 \quad 3.940366$ \\
\hline $\mathrm{H}$ & $1.202164-3.843721-2.769616$ & $\mathrm{C}$ & $-3.540281-0.884293 \quad 3.406421$ \\
\hline $\mathrm{H}$ & $0.277970-2.308596-2.845576$ & $\mathrm{C}$ & $-3.162799-1.022037 \quad 2.072285$ \\
\hline $\mathrm{H}$ & $-1.132242-4.641223 \quad 2.923684$ & $\mathrm{H}$ & $-5.151385 \quad 0.758367-0.744226$ \\
\hline $\mathrm{H}$ & $0.641579-4.823497 \quad 3.001890$ & $\mathrm{H}$ & $-6.247604 \quad 2.696633-1.824846$ \\
\hline $\mathrm{H}$ & $-0.330677-5.971442 \quad 2.058202$ & $\mathrm{H}$ & $-4.875210 \quad 4.424588-2.978657$ \\
\hline $\mathrm{Cl}$ & $0.207934 \quad 0.239349-2.833036$ & $\mathrm{H}$ & $-2.395292 \quad 4.183688-3.064268$ \\
\hline $\mathrm{N}$ & $2.179053-0.176268-0.332878$ & $\mathrm{H}$ & $-1.306940 \quad 2.207901-2.022206$ \\
\hline $\mathrm{C}$ & $2.852671-0.903134-1.237365$ & $\mathrm{H}$ & $-4.837364-1.654006 \quad 0.220222$ \\
\hline $\mathrm{C}$ & $4.239159-0.952104-1.284983$ & $\mathrm{H}$ & $-5.960382-3.489139-0.991207$ \\
\hline $\mathrm{C}$ & $4.991910-0.211527-0.366824$ & $\mathrm{H}$ & $-5.023100-4.336341-3.135034$ \\
\hline $\mathrm{C}$ & $\begin{array}{lll}4.280748 & 0.550682 & 0.565294\end{array}$ & $\mathrm{H}$ & $-2.953552-3.310710-4.068782$ \\
\hline $\mathrm{C}$ & $\begin{array}{lll}2.891968 & 0.540805 & 0.547762\end{array}$ & $\mathrm{H}$ & $-1.811047-1.468913-2.843760$ \\
\hline $\mathrm{H}$ & $2.239572-1.433734-1.965739$ & $\mathrm{H}$ & $\begin{array}{lll}-3.122406 & 2.264778 & 1.178657\end{array}$ \\
\hline $\mathrm{H}$ & $4.726408-1.538855-2.063507$ & $\mathrm{H}$ & $\begin{array}{lll}-3.776937 & 2.507833 & 3.548347\end{array}$ \\
\hline $\mathrm{C}$ & $6.474315-0.227272-0.385612$ & $\mathrm{H}$ & $-4.064062 \quad 0.492269 \quad 4.986010$ \\
\hline $\mathrm{H}$ & $\begin{array}{lll}4.799373 & 1.141532 & 1.319876\end{array}$ & $\mathrm{H}$ & $-3.644489-1.771205 \quad 4.033942$ \\
\hline $\mathrm{H}$ & $2.323302 \quad 1.137629 \quad 1.262432$ & $\mathrm{H}$ & $-2.965503-2.018093 \quad 1.665563$ \\
\hline
\end{tabular}




\section{$\mathbf{R u}(\mathrm{II})(\mathrm{Cl})\left(\mathbf{P P h}_{3}\right)-\mathrm{NNNN}$ (Cl trans to 2a)}

$\begin{array}{ccc}\mathrm{C} & 0.410642-1.886111 & 2.865372 \\ \mathrm{~N} & 0.001565-2.299399 & 1.651929 \\ \mathrm{C} & 0.433040-3.443027 & 1.121913 \\ \mathrm{C} & 1.326054-4.267606 & 1.804537 \\ \mathrm{C} & 1.714257-3.871241 & 3.089462 \\ \mathrm{C} & 1.256666-2.682780 & 3.641019 \\ \mathrm{Ru} & -0.853501-0.864189 & 0.435042 \\ \mathrm{~N} & -0.092625-3.698104 & -0.168821 \\ \mathrm{H} & 1.726645-5.171354 & 1.358779 \\ \mathrm{H} & 2.403065-4.503634 & 3.650388 \\ \mathrm{H} & 1.547456-2.330288 & 4.628947\end{array}$

$\mathrm{N} \quad-0.791585-2.685183-0.790203$

C $\quad-1.533143-3.263027-1.724801$

C $\quad-1.279219-4.658449-1.750501$

C $\quad-0.388333-4.914861-0.741422$

C $\quad-0.100075-0.572083 \quad 3.234976$

$\begin{array}{llll}\mathrm{N} & -0.825053 & 0.121573 & 2.303683\end{array}$

$\begin{array}{llll}\text { C } & -1.249639 & 1.218907 & 2.997463\end{array}$

$\begin{array}{llll}\text { C } & -0.684686 & 1.137710 & 4.307634\end{array}$

N $\quad 0.041308-0.015006 \quad 4.432015$

$\begin{array}{llll}\text { C } & -0.938572 & 2.147983 & 5.256727\end{array}$

$\begin{array}{llll}\text { C } & -1.762798 & 3.194628 & 4.891275\end{array}$

$\begin{array}{llll}\text { C } & -2.359021 & 3.246318 & 3.605424\end{array}$

$\begin{array}{llll}\text { C } & -2.123252 & 2.270361 & 2.654551\end{array}$

C $\quad-2.585364-2.538454-2.497116$

H $\quad-1.736901-5.391351-2.407024$

C $\quad 0.136343-6.232786-0.279891$

$\begin{array}{llll}\text { H } & -0.499988 & 2.080278 & 6.252898\end{array}$

$\begin{array}{llll}\mathrm{H} & -1.982402 & 3.986769 & 5.608440\end{array}$

$\mathrm{H} \quad-3.039775 \quad 4.065707 \quad 3.369434$

$\mathrm{H} \quad-2.628875 \quad 2.288968 \quad 1.688237$

$\mathrm{H} \quad-3.416319-3.234153-2.675725$

$\mathrm{H} \quad-2.227130-2.170026-3.467543$

$\mathrm{H} \quad-2.960280-1.708935-1.885603$

H $\quad-0.095214-6.407835 \quad 0.780247$

$\mathrm{H} \quad 1.223523-6.318475-0.418068$

H $\quad-0.343913-7.020546-0.871599$

$\mathrm{Cl} \quad-3.112427-1.689243 \quad 0.925621$

$\mathrm{N} \quad 1.227038-0.331170 \quad 0.177042$

C $\quad 2.023603-1.011451-0.665022$

C $\quad 3.346023-0.669582-0.908658$

C $\quad 3.910705 \quad 0.425796-0.244501$

$\begin{array}{llll}\text { C } & 3.097063 & 1.090464 & 0.676567\end{array}$
C

$\mathrm{H}$

$\mathrm{H}$

$\mathrm{C}$

$\mathrm{H}$

$\mathrm{H}$

$\mathrm{C}$

C

$\mathrm{C}$

C

$\mathrm{C}$

$\mathrm{H}$

$\mathrm{H}$

$\mathrm{H}$

$\mathrm{H}$

$\mathrm{H}$

$\mathrm{P}$

\section{C}

C

C

C

C

C

C

C

C

C

C

C

C

C

C

C

C

C

$\mathrm{H}$

$\mathrm{H}$

$\mathrm{H}$

$\mathrm{H}$

$\mathrm{H}$

$\mathrm{H}$

$\mathrm{H}$

$\mathrm{H}$

H $\begin{array}{lll}1.781220 & 0.686734 & 0.859367\end{array}$

$1.559748-1.855953-1.178049$

$3.918216-1.237168-1.642412$

$\begin{array}{llll}5.298145 & 0.868854 & -0.513831\end{array}$

$\begin{array}{lll}3.477652 & 1.925972 & 1.263427\end{array}$

$\begin{array}{llll}1.129324 & 1.200751 & 1.564100\end{array}$

$6.314442-0.060579-0.769755$

$7.614543 \quad 0.366489-1.025865$

$7.914970 \quad 1.728135-1.036607$

$6.909006 \quad 2.660792-0.786564$

$5.609743 \quad 2.234947-0.523863$

$6.089307-1.127985-0.739851$

$8.398837-0.367976-1.211518$

\begin{tabular}{ll}
$8.933037 \quad 2.062163-1.238853$ \\
\hline
\end{tabular}

$\begin{array}{lll}7.136462 & 3.727094-0.800506\end{array}$

$\begin{array}{lll}4.817387 & 2.965618-0.350338\end{array}$

$-1.385500 \quad 0.895189-1.064168$

$-0.068834 \quad 2.216703-1.045581$

$\begin{array}{lll}-2.916231 & 1.917156-0.961684\end{array}$

$-1.3736190 .415848-2.856975$

$0.960994 \quad 2.295590-1.989051$

$1.981839 \quad 3.239132-1.850753$

$1.981891 \quad 4.126004-0.776577$

$\begin{array}{lll}0.951404 & 4.065067 & 0.161920\end{array}$

$\begin{array}{llll}-0.060291 & 3.117019 & 0.030437\end{array}$

$\begin{array}{ll}-2.393276 & 0.765634-3.747472\end{array}$

$-2.348705 \quad 0.336948-5.075580$

$-1.278820-0.427687-5.535976$

$-0.253953-0.778019-4.655847$

$-0.313018-0.373343-3.324851$

$\begin{array}{lll}-2.961197 & 3.226421-1.464080\end{array}$

$-4.143369 \quad 3.959352-1.402341$

$\begin{array}{lll}-5.290250 & 3.391753-0.843527\end{array}$

$\begin{array}{ll}-5.250453 & 2.090037-0.350460\end{array}$

$\begin{array}{lll}-4.068309 & 1.348662-0.406218\end{array}$

$0.977656 \quad 1.629674-2.850693$

$2.776831 \quad 3.280479-2.597064$

$2.774059 \quad 4.870401-0.676980$

$\begin{array}{llll}0.930051 & 4.754889 & 1.006586\end{array}$

$\begin{array}{llll}-0.848750 & 3.080470 & 0.782053\end{array}$

$\begin{array}{lll}-3.238552 & 1.363401 & -3.405033\end{array}$

$-3.1583120 .608164-5.754404$

$-1.244779-0.754138-6.576137$

$0.585238-1.382772-5.002538$ 


\begin{tabular}{|c|c|c|c|c|c|c|}
\hline $\mathrm{H}$ & \multicolumn{3}{|c|}{$0.473184-0.678417-2.633677$} & \multirow{2}{*}{$\begin{array}{c}\mathrm{H} \\
\mathrm{Cl}\end{array}$} & \multirow{2}{*}{$\begin{array}{c}-4.165234-4.990061 \\
0.032184-0.164175\end{array}$} & \multirow{2}{*}{$\begin{array}{l}2.494133 \\
3.422616\end{array}$} \\
\hline $\mathrm{H}$ & -2.068318 & 3.676232 & -1.902284 & & & \\
\hline $\mathrm{H}$ & -4.168673 & 4.978804 & -1.789646 & $\mathrm{~N}$ & $1.821038-0.346045$ & 0.681703 \\
\hline $\mathrm{H}$ & -6.213729 & 3.970295 & -0.790248 & $\mathrm{C}$ & $2.573866-0.972018$ & 1.606623 \\
\hline $\mathrm{H}$ & -6.140267 & 1.642790 & 0.093906 & $\mathrm{C}$ & $3.954887-1.085687$ & 1.523564 \\
\hline \multirow[t]{2}{*}{$\mathrm{H}$} & -4.031216 & 0.336555 & 0.004333 & $\mathrm{C}$ & $4.640609-0.516103$ & 0.446062 \\
\hline & & & & $\mathrm{C}$ & $3.861027 \quad 0.164163$ & -0.492125 \\
\hline \multicolumn{4}{|c|}{$\mathrm{Ru}(\mathrm{II})(\mathrm{Cl})\left(\mathrm{PPh}_{3}\right)-\mathrm{NNNN}(\mathrm{NNN}$ trans to $2 \mathrm{a})$} & $\mathrm{C}$ & $2.480497 \quad 0.226706$ & -0.336141 \\
\hline $\mathrm{C}$ & -2.742561 & 1.338667 & 1.579239 & $\mathrm{H}$ & $2.025778-1.381571$ & 2.453036 \\
\hline $\mathrm{N}$ & -2.299577 & 0.070315 & 1.467891 & $\mathrm{H}$ & $4.487290-1.640104$ & 2.296530 \\
\hline $\mathrm{C}$ & -3.073831 & 0.979494 & 1.750057 & $\mathrm{C}$ & $6.111844-0.624268$ & 0.307915 \\
\hline $\mathrm{C}$ & -4.417878 & 0.811236 & 2.075161 & $\mathrm{H}$ & $4.319864 \quad 0.677614$ & -1.337219 \\
\hline $\mathrm{C}$ & -4.903050 & 0.500489 & 2.144909 & $\mathrm{H}$ & $1.917950 \quad 0.791254$ & -1.081643 \\
\hline $\mathrm{C}$ & -4.074485 & 1.589013 & 1.917346 & $\mathrm{C}$ & $6.940803-0.575305$ & 1.436345 \\
\hline $\mathrm{Ru}$ & -0.372104 & 0.194261 & 0.975532 & $\mathrm{C}$ & $8.323119-0.675521$ & 1.302354 \\
\hline $\mathrm{N}$ & -2.368976 & -2.209364 & 1.697220 & $\mathrm{C}$ & $8.895671-0.832056$ & 0.040529 \\
\hline $\mathrm{H}$ & -5.077172 & 1.645002 & 2.279761 & $\mathrm{C}$ & $8.078515-0.884093-$ & -1.088174 \\
\hline $\mathrm{H}$ & -5.951070 & 0.657272 & 2.401536 & $\mathrm{C}$ & $6.696668-0.777707$ - & -0.956044 \\
\hline $\mathrm{H}$ & -4.407335 & 2.622320 & 1.995981 & $\mathrm{H}$ & $6.499554-0.428200$ & 2.423493 \\
\hline $\mathrm{N}$ & -1.035892 & -2.180495 & 1.384826 & $\mathrm{H}$ & $8.956803-0.623833$ & 2.188266 \\
\hline $\mathrm{C}$ & -0.598544 & 3.422670 & 1.483721 & $\mathrm{H}$ & $9.978257-0.911919$ & -0.063287 \\
\hline $\mathrm{C}$ & -1.659388 & 4.284261 & 1.853087 & $\mathrm{H}$ & $8.519369-1.012965$ & -2.077294 \\
\hline $\mathrm{C}$ & -2.773575 & 3.488665 & 1.986344 & $\mathrm{H}$ & $6.058737-0.839328$ & 1.839542 \\
\hline $\mathrm{C}$ & -1.718176 & 2.343389 & 1.330516 & $\mathrm{P}$ & $-1.010060-0.145092-$ & 1.289101 \\
\hline $\mathrm{N}$ & -0.449159 & 1.915377 & 1.060467 & $\mathrm{C}$ & $0.229203-0.541259$ & -2.613881 \\
\hline $\mathrm{C}$ & 0.251690 & 3.079615 & 0.936202 & $\mathrm{C}$ & $-1.692228 \quad 1.510052$ & -1.729317 \\
\hline $\mathrm{C}$ & -0.677373 & 4.152872 & 1.115240 & $\mathrm{C}$ & $-2.358865-1.257574-$ & -1.958632 \\
\hline $\mathrm{N}$ & -1.924051 & 3.655166 & 1.369923 & $\mathrm{C}$ & $0.295611 \quad 0.152799$ & -3.826919 \\
\hline $\mathrm{C}$ & -0.254562 & 5.490721 & 0.996399 & $\mathrm{C}$ & $1.204843-0.234268-$ & -4.813051 \\
\hline $\mathrm{C}$ & 1.075857 & 5.733672 & 0.710481 & $\mathrm{C}$ & $2.043816-1.326132-$ & -4.605284 \\
\hline $\mathrm{C}$ & 1.999885 & 4.668873 & 0.561650 & $\mathrm{C}$ & $1.970342-2.033219$ & -3.405761 \\
\hline $\mathrm{C}$ & 1.611195 & 3.346203 & 0.676989 & $\mathrm{C}$ & $1.069363-1.644811-$ & -2.416830 \\
\hline $\mathrm{C}$ & 0.824536 & -3.788302 & 1.229643 & $\mathrm{C}$ & $-3.045846-0.911772$ & 3.132377 \\
\hline $\mathrm{H}$ & -1.613007 & -5.356495 & 2.014435 & $\mathrm{C}$ & $-3.994214-1.767548$ & -3.685089 \\
\hline $\mathrm{C}$ & -4.159070 & 3.903734 & 2.349259 & $\mathrm{C}$ & $-4.268206-2.997205-$ & -3.086268 \\
\hline $\mathrm{H}$ & -0.971359 & 6.301752 & 1.131713 & $\mathrm{C}$ & $-3.568323-3.368443-$ & -1.942509 \\
\hline $\mathrm{H}$ & 1.431652 & 6.760760 & 0.615538 & $\mathrm{C}$ & $-2.618855-2.508059-$ & -1.386879 \\
\hline $\mathrm{H}$ & 3.048165 & 4.903030 & 0.369203 & $\mathrm{C}$ & $-0.835335 \quad 2.592752$ & -1.956656 \\
\hline $\mathrm{H}$ & 2.340727 & 2.540873 & 0.606341 & $\mathrm{C}$ & $-1.342190 \quad 3.881725$ & -2.100894 \\
\hline $\mathrm{H}$ & 0.897122 & -4.827067 & 0.888060 & $\mathrm{C}$ & $-2.714765 \quad 4.106439$ & -2.014378 \\
\hline $\mathrm{H}$ & 1.416824 & -3.670802 & 2.148182 & $\mathrm{C}$ & $-3.576250 \quad 3.037679$ & -1.779046 \\
\hline $\mathrm{H}$ & 1.242872 & -3.119270 & 0.466798 & $\mathrm{C}$ & $-3.069539 \quad 1.748763$ & -1.629703 \\
\hline $\mathrm{H}$ & -4.874114 & 3.658159 & 1.551192 & $\mathrm{H}$ & $-0.358177 \quad 1.006798$ & -4.006827 \\
\hline $\mathrm{H}$ & -4.494242 & -3.432760 & 3.283778 & $\mathrm{H}$ & $1.252019 \quad 0.320202$ & -5.751335 \\
\hline
\end{tabular}



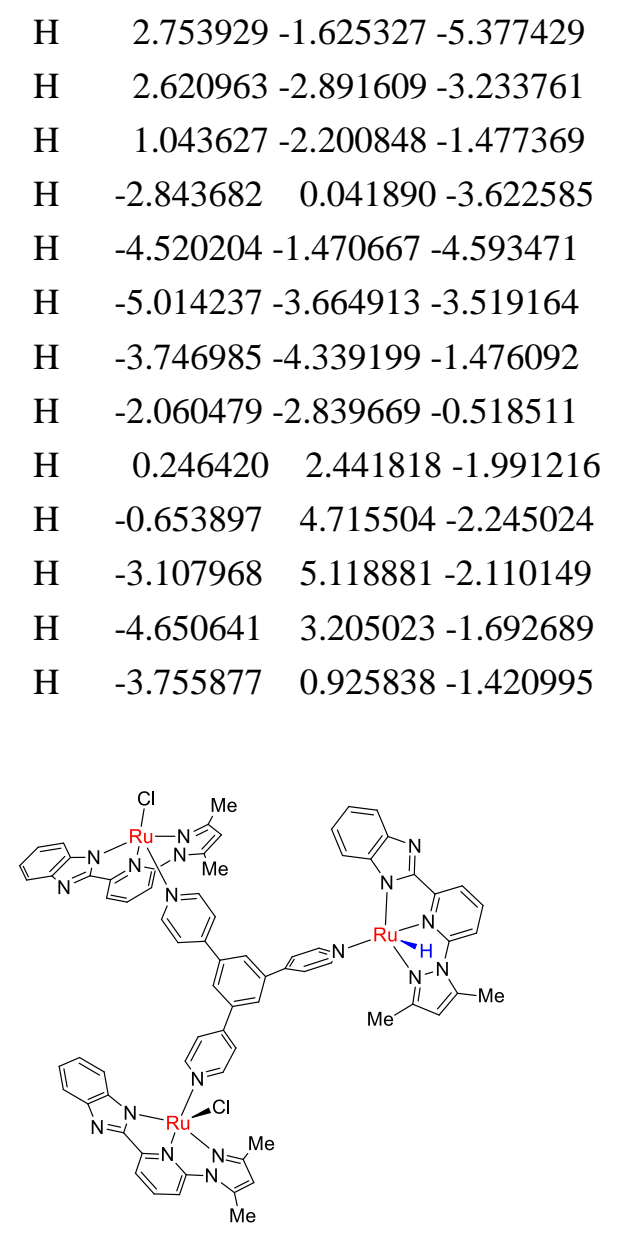

\section{Complex 3'}

$\begin{array}{ccc}\mathrm{C} & 7.059240-4.677459 & 1.691076 \\ \mathrm{~N} & 6.080184-5.507893 & 1.258047 \\ \mathrm{C} & 5.427425-6.325290 & 2.093282 \\ \mathrm{C} & 5.727314-6.349574 & 3.454221 \\ \mathrm{C} & 6.731040-5.486348 & 3.911720 \\ \mathrm{C} & 7.408138-4.642398 & 3.043249 \\ \mathrm{Ru} & 5.670251-5.508292 & -0.714875 \\ \mathrm{~N} & 4.460679-7.127901 & 1.436891 \\ \mathrm{H} & 5.220457-7.009135 & 4.147453 \\ \mathrm{H} & 6.981110-5.490802 & 4.972900 \\ \mathrm{H} & 8.196333-3.962525 & 3.362012 \\ \mathrm{~N} & 4.363708-7.043631 & 0.067067 \\ \mathrm{C} & 3.435243-7.911561 & -0.304314 \\ \mathrm{C} & 2.913738-8.573198 & 0.835845 \\ \mathrm{C} & 3.578836-8.062773 & 1.924510 \\ \mathrm{C} & 7.640413-3.874153 & 0.626032 \\ \mathrm{~N} & 7.171615-4.077116 & -0.648087 \\ \mathrm{C} & 7.857810-3.160464 & -1.389673 \\ \mathrm{C} & 8.715485-2.453127 & -0.493788 \\ \mathrm{~N} & 8.555609-2.924177 & 0.782565\end{array}$

C $\quad 9.554228-1.429358-0.982026$

C $\quad 9.507034-1.146382-2.332014$

C $\quad 8.647856-1.857542-3.212973$

C $\quad 7.817708-2.866793-2.766802$

C $\quad 3.068463-8.094656-1.738894$

H $\quad 2.143707-9.337618 \quad 0.857463$

C $\quad 3.399985-8.426930 \quad 3.360518$

$\mathrm{H} \quad 10.211604-0.888115-0.301046$

H $\quad 10.142334-0.358771-2.739529$

H $\quad 8.649540-1.595852-4.272091$

H $\quad 7.158030-3.428974-3.429160$

H $\quad 2.024847-7.789539-1.907499$

H $\quad 3.149853-9.155215-2.014763$

H $\quad 3.731546-7.502263-2.384977$

H $\quad 3.138509-7.553702 \quad 3.974086$

H $\quad 4.303346-8.892116 \quad 3.779530$

$\mathrm{H} \quad 2.581832-9.152670 \quad 3.430915$

Cl $\quad 5.362978-5.686645-3.112028$

$\begin{array}{llll}\text { C } & 2.884377 & 7.285107 & 2.307071\end{array}$

$\begin{array}{llll}\mathrm{N} & 1.639501 & 7.384422 & 1.782201\end{array}$

$\begin{array}{llll}\text { C } & 0.565311 & 7.606305 & 2.549836\end{array}$

$\begin{array}{llll}\text { C } & 0.687194 & 7.742099 & 3.931939\end{array}$

$\begin{array}{llll}\text { C } & 1.969737 & 7.631614 & 4.483229\end{array}$

$\begin{array}{llll}\text { C } & 3.081191 & 7.401859 & 3.684808\end{array}$

$\mathrm{Ru} \quad 1.460928 \quad 7.231012-0.218969$

$\begin{array}{llll}\mathrm{N} & -0.636707 & 7.696365 & 1.805329\end{array}$

$\mathrm{H} \quad-0.164993 \quad 7.934032 \quad 4.571911$

$\begin{array}{llll}\mathrm{H} & 2.086059 & 7.735723 & 5.562272\end{array}$

$\begin{array}{llll}\mathrm{H} & 4.092705 & 7.314552 & 4.077534\end{array}$

$\begin{array}{llll}\mathrm{N} & -0.560870 & 7.636156 & 0.432449\end{array}$

C $\quad-1.795002 \quad 7.782426-0.024891$

$\begin{array}{llll}\text { C } & -2.693794 & 7.937666 & 1.060531\end{array}$

$\begin{array}{llll}\text { C } & -1.938749 & 7.881850 & 2.206763\end{array}$

$\begin{array}{llll}\text { C } & 3.910489 & 7.039965 & 1.303959\end{array}$

N $\quad 3.512670 \quad 6.985664-0.008319$

C $\quad 4.663333 \quad 6.688251-0.678060$

$\begin{array}{llll}\text { C } & 5.701559 & 6.585015 & 0.296376\end{array}$

$\begin{array}{llll}\mathrm{N} & 5.195633 & 6.809629 & 1.549709\end{array}$

C $\quad 7.018321 \quad 6.279889-0.107093$

C $\quad 7.251402 \quad 6.085656-1.453646$

C $\quad 6.208168 \quad 6.189192-2.413167$

C $\quad 4.910061 \quad 6.490188-2.050645$

C $\quad-2.092637 \quad 7.774357-1.486831$

$\begin{array}{llll}\mathrm{H} & -3.769079 & 8.074855 & 1.008187\end{array}$ 


\begin{tabular}{|c|c|c|c|c|}
\hline $\mathrm{C}$ & -2.408400 & $7.991666 \quad 3.618905$ & $\mathrm{C}$ & $\begin{array}{ll}-5.427983 \quad 2.007055 & -2.648981\end{array}$ \\
\hline $\mathrm{H}$ & 7.814557 & $6.202627 \quad 0.633861$ & $\mathrm{C}$ & $-5.772167 \quad 0.861672-1.953211$ \\
\hline $\mathrm{H}$ & 8.259007 & $5.846629-1.796670$ & $\mathrm{C}$ & $\begin{array}{lll}-6.254901 & -5.055485 & 1.965877\end{array}$ \\
\hline $\mathrm{H}$ & 6.445407 & $6.027113-3.465612$ & $\mathrm{H}$ & $-8.686750-6.760816 \quad 2.613504$ \\
\hline $\mathrm{H}$ & 4.097408 & $6.581475-2.772674$ & $\mathrm{C}$ & $\begin{array}{lll}-11.318376 & -5.798743 \quad 1.683056\end{array}$ \\
\hline $\mathrm{H}$ & -2.637492 & $8.687741-1.763282$ & $\mathrm{H}$ & $-8.523051 \quad 3.258510-3.475823$ \\
\hline $\mathrm{H}$ & -2.736697 & $6.918759-1.739316$ & $\mathrm{H}$ & $-6.089889 \quad 3.763432-3.732784$ \\
\hline $\mathrm{H}$ & -1.162885 & $7.719818-2.070302$ & $\mathrm{H}$ & $-4.373500 \quad 2.245865-2.800363$ \\
\hline $\mathrm{H}$ & -2.009249 & $8.888273 \quad 4.113756$ & $\mathrm{H}$ & $-5.008845 \quad 0.187721-1.559638$ \\
\hline $\mathrm{H}$ & -2.129086 & $7.109887 \quad 4.212061$ & $\mathrm{H}$ & $\begin{array}{lll}-5.812038 & -5.787819 & 1.274452\end{array}$ \\
\hline $\mathrm{H}$ & -3.501666 & $8.067207 \quad 3.611448$ & $\mathrm{H}$ & $-6.052132-5.392269 \quad 2.991274$ \\
\hline $\mathrm{Cl}$ & 1.289038 & $7.291295-2.632763$ & $\mathrm{H}$ & $\begin{array}{lll}-5.771501 & -4.084922 & 1.797022\end{array}$ \\
\hline $\mathrm{N}$ & 1.100098 & $5.259571-0.116132$ & $\mathrm{H}$ & $-11.776284-5.902599 \quad 0.689502$ \\
\hline $\mathrm{C}$ & 1.887462 & $4.404819 \quad 0.563199$ & $\mathrm{H}$ & $-11.967515-5.162970 \quad 2.301994$ \\
\hline $\mathrm{C}$ & 1.659804 & $3.036209 \quad 0.578232$ & $\mathrm{H}$ & $-11.275862-6.791299 \quad 2.146057$ \\
\hline $\mathrm{C}$ & 0.577499 & $2.501220-0.125445$ & $\mathrm{H}$ & $-7.417933-3.138996-1.229210$ \\
\hline $\mathrm{C}$ & -0.224915 & $3.398300-0.838209$ & $\mathrm{~N}$ & $\begin{array}{lll}-5.453242 & -1.986775 & 0.123859\end{array}$ \\
\hline $\mathrm{C}$ & 0.066567 & $4.753061-0.817479$ & $\mathrm{C}$ & $-4.965971-1.021073 \quad 0.924807$ \\
\hline $\mathrm{H}$ & 2.729444 & $\begin{array}{ll}4.842280 & 1.098402\end{array}$ & $\mathrm{C}$ & $-3.642137-0.609493 \quad 0.908049$ \\
\hline $\mathrm{H}$ & 2.324191 & $2.398435 \quad 1.160842$ & $\mathrm{C}$ & $-2.742187-1.217470 \quad 0.025573$ \\
\hline $\mathrm{H}$ & -1.060659 & $3.047183-1.443277$ & $\mathrm{C}$ & $-3.240407-2.246302-0.780106$ \\
\hline $\mathrm{H}$ & -0.506932 & $5.474786-1.397126$ & $\mathrm{C}$ & $-4.582920-2.595733-0.702100$ \\
\hline $\mathrm{C}$ & -10.109897 & $-1.103832-0.897210$ & $\mathrm{H}$ & $\begin{array}{l}-5.690969-0.541124 \quad 1.585812 \\
\end{array}$ \\
\hline $\mathrm{N}$ & $-9.566663-2$ & $2.151441-0.236213$ & $\mathrm{H}$ & $\begin{array}{lll}-3.317096 & 0.183231 & 1.581814\end{array}$ \\
\hline $\mathrm{C}$ & -10.332093 & $-3.093870 \quad 0.323290$ & $\mathrm{C}$ & $-1.333411-0.766597-0.066492$ \\
\hline $\mathrm{C}$ & -11.723011 & $-3.044532 \quad 0.240860$ & $\mathrm{H}$ & $-2.598925-2.748300-1.504833$ \\
\hline $\mathrm{C}$ & -12.289391 & $-1.973113-0.457920$ & $\mathrm{H}$ & $-5.008464-3.367948-1.342133$ \\
\hline $\mathrm{C}$ & -11.493287 & $-0.990361-1.033120$ & $\mathrm{C}$ & $-1.035600 \quad 0.599271-0.046814$ \\
\hline $\mathrm{Ru}$ & $-7.562566-2$ & $2.192058-0.039825$ & $\mathrm{C}$ & $0.286951 \quad 1.047104-0.120030$ \\
\hline $\mathrm{N}$ & $-9.566996-$ & $-4.082215 \quad 0.991434$ & $\mathrm{C}$ & $1.321177 \quad 0.108757-0.203513$ \\
\hline $\mathrm{H}$ & -12.356822 & $-3.792270 \quad 0.702560$ & $\mathrm{C}$ & $1.042818-1.262604-0.233209$ \\
\hline $\mathrm{H}$ & -13.374898 & $-1.913475-0.539080$ & $\mathrm{C}$ & $-0.288480-1.689291-0.170039$ \\
\hline $\mathrm{H}$ & -11.896667 & $-0.137591-1.576370$ & $\mathrm{H}$ & $\begin{array}{lll}-1.848969 & 1.324754 & 0.019439\end{array}$ \\
\hline $\mathrm{N}$ & $-8.211523-$ & $3.890335 \quad 1.073819$ & $\mathrm{H}$ & $2.352020 \quad 0.453312-0.306365$ \\
\hline $\mathrm{C}$ & $-7.722214-4$ & $4.931795 \quad 1.727323$ & $\mathrm{C}$ & $2.140711-2.254805-0.329148$ \\
\hline $\mathrm{C}$ & $-8.768557-$ & $5.820316 \quad 2.078234$ & $\mathrm{H}$ & $-0.513047-2.757453-0.150627$ \\
\hline $\mathrm{C}$ & $-9.929330-$ & $5.260875 \quad 1.597025$ & $\mathrm{C}$ & $3.365704-2.058685 \quad 0.314980$ \\
\hline $\mathrm{C}$ & $-9.115094-($ & $0.165092-1.410523$ & $\mathrm{C}$ & $4.365781-3.014781 \quad 0.211485$ \\
\hline $\mathrm{N}$ & $-7.794978-($ & $0.447732-1.179012$ & $\mathrm{~N}$ & $4.208738-4.150803-0.492912$ \\
\hline $\mathrm{C}$ & -7.143699 & $0.582974-1.794618$ & $\mathrm{C}$ & $3.038691-4.344762-1.133475$ \\
\hline $\mathrm{C}$ & -8.139178 & $1.443174-2.351703$ & $\mathrm{C}$ & $1.997570-3.432309-1.071374$ \\
\hline $\mathrm{N}$ & -9.387088 & $0.940371-2.093311$ & $\mathrm{H}$ & $3.550180-1.170488 \quad 0.918538$ \\
\hline $\mathrm{C}$ & -7.760378 & $2.604387-3.051816$ & $\mathrm{H}$ & $5.331765-2.873300 \quad 0.694824$ \\
\hline $\mathrm{C}$ & -6.410841 & $2.874055-3.188644$ & $\mathrm{H}$ & $2.978813-5.249411-1.736998$ \\
\hline
\end{tabular}




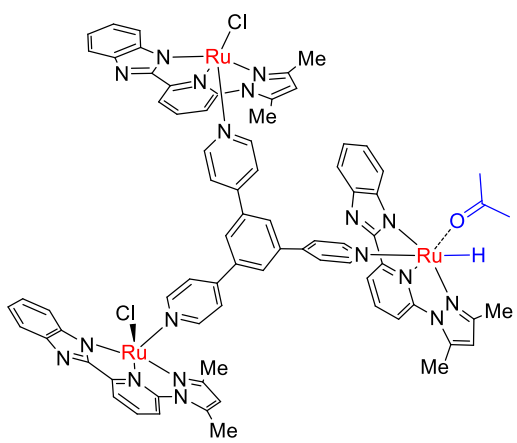

\section{Complex 3"}

C $\quad-6.601162-5.162104-1.916465$

N $\quad-5.535607-5.896792-1.515699$

C $\quad-4.770618-6.564491-2.388011$

C $\quad-5.038487-6.524242-3.755232$

C $\quad-6.132222-5.758473-4.178667$

C $\quad-6.925397-5.070076-3.272062$

$\begin{array}{llll}\mathrm{Ru} & -5.168109 & -5.994146 & 0.462869\end{array}$

N $\quad-3.724931-7.289337-1.762930$

$\mathrm{H} \quad-4.440303-7.063617-4.478956$

$\mathrm{H} \quad-6.358825-5.714494-5.244194$

H $\quad-7.785253-4.469669-3.564046$

N $\quad-3.667494-7.297378-0.388405$

C $\quad-2.645309-8.069403-0.053411$

C $\quad-2.021703-8.573618-1.222350$

C $\quad-2.722961-8.068333-2.290597$

C $\quad-7.296967-4.515409-0.814072$

$\begin{array}{llll}\text { N } & -6.830442 & -4.752348 & 0.455007\end{array}$

$\begin{array}{llll}\text { C } & -7.639513 & -3.984430 & 1.240158\end{array}$

C $\quad-8.561896-3.324179 \quad 0.373221$

$\mathrm{N} \quad-8.320584-3.676626-0.928333$

C $\quad-9.531226-2.450866 \quad 0.909533$

C $\quad-9.545435-2.265213 \quad 2.277096$

C $\quad-8.620620-2.927935 \quad 3.128876$

C $\quad-7.662243-3.790851 \quad 2.635199$

C $\quad-2.287980-8.313466 \quad 1.374469$

H $\quad-1.161394-9.232910-1.276518$

C $\quad-2.469954-8.297152-3.743307$

H $\quad-10.238237-1.9459690 .250700$

H $\quad-10.282139-1.594697 \quad 2.721710$

H $\quad-8.675316-2.747910 \quad 4.203512$

H $\quad-6.949417-4.314777 \quad 3.273419$

H $\quad-1.294700-7.894864 \quad 1.595479$
H $\quad-2.241193-9.393743 \quad 1.570338$

H $\quad-3.033396-7.858276 \quad 2.041670$

H $\quad-2.308661-7.353963-4.283412$

H $\quad-3.298278-8.838333-4.221839$

H $\quad-1.565102-8.907514-3.841865$

$\mathrm{Cl} \quad-4.893952-6.315072 \quad 2.851137$

C $\quad-3.867084 \quad 7.063528-2.099969$

$\mathrm{N} \quad-2.644206 \quad 7.291093-1.563601$

C $\quad-1.5849527 .594284-2.324200$

C $\quad-1.700907 \quad 7.690376-3.710027$

C $\quad-2.960266 \quad 7.448954-4.272944$

C $\quad-4.055625 \quad 7.132015-3.482068$

$\begin{array}{llll}\mathrm{Ru} & -2.479267 & 7.203704 & 0.442710\end{array}$

N $\quad-0.405914 \quad 7.809319-1.568780$

$\mathrm{H} \quad-0.862098 \quad 7.949340-4.344101$

$\mathrm{H} \quad-3.071781 \quad 7.519789-5.355161$

$\mathrm{H} \quad-5.049142 \quad 6.941441-3.884194$

$\begin{array}{llll}\mathrm{N} & -0.497339 & 7.788570 & -0.195936\end{array}$

$\begin{array}{llll}\text { C } & 0.713868 & 8.050762 & 0.270791\end{array}$

C $\quad 1.613488 \quad 8.241540-0.808044$

C $\quad 0.882866 \quad 8.084838-1.960908$

C $\quad-4.878336 \quad 6.743034-1.102876$

$\begin{array}{llll}\mathrm{N} & -4.492786 & 6.750265 & 0.214293\end{array}$

$\begin{array}{llll}\text { C } & -5.620417 & 6.364925 & 0.878315\end{array}$

C $\quad-6.633592 \quad 6.150113-0.104249$

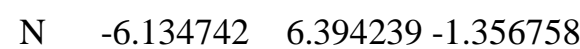

$\begin{array}{llll}\text { C } & -7.922886 & 5.737812 & 0.292268\end{array}$

$\begin{array}{llll}\text { C } & -8.154364 & 5.551824 & 1.640321\end{array}$

$\begin{array}{llll}\text { C } & -7.136124 & 5.767319 & 2.608004\end{array}$

$\begin{array}{llll}\text { C } & -5.865359 & 6.174509 & 2.252199\end{array}$

$\begin{array}{llll}\text { C } & 0.987880 & 8.117434 & 1.735887\end{array}$

H $\quad 2.673617 \quad 8.465789-0.748203$

C $\quad 1.364078 \quad 8.178008-3.370303$

$\mathrm{H} \quad-8.700168 \quad 5.575076-0.454922$

$\begin{array}{llll}\mathrm{H} & -9.141127 & 5.231863 & 1.977997\end{array}$

$\begin{array}{llll}\mathrm{H} & -7.371038 & 5.607283 & 3.661311\end{array}$

$\begin{array}{llll}\mathrm{H} & -5.072797 & 6.353046 & 2.980198\end{array}$

$\mathrm{H} \quad 1.442534 \quad 9.085513 \quad 1.988430$

$\begin{array}{llll}\mathrm{H} & 1.702919 & 7.332904 & 2.025275\end{array}$

$\begin{array}{llll}\mathrm{H} & 0.057810 & 7.996416 & 2.308795\end{array}$

$\mathrm{H} \quad 0.914128 \quad 9.029107-3.900691$

$\mathrm{H} \quad 1.151653 \quad 7.259875-3.935216$

$\mathrm{H} \quad 2.449853 \quad 8.325344-3.352818$

$\begin{array}{llll}\mathrm{Cl} & -2.347062 & 7.338790 & 2.858813\end{array}$ 


\begin{tabular}{|c|c|c|c|c|}
\hline $\mathrm{N}$ & -1.914720 & $5.279613 \quad 0.384480$ & $\mathrm{H}$ & $8.894745-6.487291-1.811572$ \\
\hline $\mathrm{C}$ & -2.578122 & $4.341632-0.316706$ & $\mathrm{H}$ & $7.150312-6.399941-2.174197$ \\
\hline $\mathrm{C}$ & -2.193895 & $3.008359-0.324379$ & $\mathrm{H}$ & $7.752326-7.543179-0.953350$ \\
\hline $\mathrm{C}$ & -1.080455 & $2.598057 \quad 0.414001$ & $\mathrm{H}$ & $9.303019-1.604649 \quad 0.881863$ \\
\hline $\mathrm{C}$ & -0.414533 & $3.579093 \quad 1.156622$ & $\mathrm{O}$ & $7.438515-0.774965 \quad 2.705647$ \\
\hline $\mathrm{C}$ & -0.856118 & $4.891977 \quad 1.123209$ & $\mathrm{C}$ & $8.180033-0.078616 \quad 3.391695$ \\
\hline $\mathrm{H}$ & -3.446745 & $4.681739-0.879475$ & $\mathrm{C}$ & $\begin{array}{lll}7.743892 & 0.329836 & 4.770284\end{array}$ \\
\hline $\mathrm{H}$ & -2.759536 & $2.302031-0.931491$ & $\mathrm{C}$ & $\begin{array}{lll}9.522964 & 0.378059 & 2.907070\end{array}$ \\
\hline $\mathrm{H}$ & 0.433808 & $3.324464 \quad 1.791329$ & $\mathrm{H}$ & $\begin{array}{lll}7.638110 & 1.425750 & 4.799155\end{array}$ \\
\hline $\mathrm{H}$ & -0.388848 & $5.673730 \quad 1.720216$ & $\mathrm{H}$ & $\begin{array}{lll}8.522328 & 0.066462 \quad 5.501871\end{array}$ \\
\hline $\mathrm{C}$ & 8.297750 & $-1.112858-2.161961$ & $\mathrm{H}$ & $6.791650-0.143894 \quad 5.031353$ \\
\hline $\mathrm{N}$ & 8.062324 & $-2.051958-1.213885$ & $\mathrm{H}$ & $9.910467 \quad 1.207159 \quad 3.512293$ \\
\hline $\mathrm{C}$ & 8.068753 & $-3.356550-1.526286$ & $\mathrm{H}$ & $\begin{array}{lll}9.467517 & 0.642396 & 1.842559\end{array}$ \\
\hline $\mathrm{C}$ & 8.319480 & $-3.794946-2.825321$ & $\mathrm{H}$ & $10.210333-0.480710 \quad 2.977540$ \\
\hline $\mathrm{C}$ & 8.562487 & $-2.827375-3.804468$ & $\mathrm{~N}$ & $5.447219-1.171271 \quad 0.438119$ \\
\hline $\mathrm{C}$ & 8.551344 & $-1.475668-3.483108$ & $\mathrm{C}$ & $4.922959-0.184584-0.301956$ \\
\hline $\mathrm{Ru}$ & 7.757974 & $-1.466740 \quad 0.647998$ & $\mathrm{C}$ & $3.560141 \quad 0.089032-0.343374$ \\
\hline $\mathrm{N}$ & 7.802825 & $-4.180537-0.406003$ & $\mathrm{C}$ & $2.676568-0.686745 \quad 0.412790$ \\
\hline $\mathrm{H}$ & 8.332241 & $-4.846973-3.083877$ & $\mathrm{C}$ & $3.226756-1.723870 \quad 1.173581$ \\
\hline $\mathrm{H}$ & 8.762384 & $-3.147982-4.826821$ & $\mathrm{C}$ & $4.602462-1.923878 \quad 1.154216$ \\
\hline $\mathrm{H}$ & 8.731448 & $-0.687301-4.212026$ & $\mathrm{H}$ & $5.633379 \quad 0.412508-0.877121$ \\
\hline $\mathrm{N}$ & 7.592721 & -3.5616890 .798588 & $\mathrm{H}$ & $3.195189 \quad 0.896234-0.978777$ \\
\hline $\mathrm{C}$ & 7.371143 & $-4.519860 \quad 1.684896$ & $\mathrm{C}$ & $\begin{array}{lll}1.218972 & -0.409947 & 0.418132\end{array}$ \\
\hline $\mathrm{C}$ & 7.434186 & $\begin{array}{ll}-5.786508 & 1.052258\end{array}$ & $\mathrm{H}$ & $2.596253-2.351943 \quad 1.803651$ \\
\hline $\mathrm{C}$ & 7.711998 & $-5.545728-0.272849$ & $\mathrm{H}$ & $5.062819-2.718214 \quad 1.746397$ \\
\hline $\mathrm{C}$ & 8.226991 & $0.253777-1.651991$ & $\mathrm{C}$ & $\begin{array}{lll}0.754440 & 0.908433 & 0.412086\end{array}$ \\
\hline $\mathrm{N}$ & 7.993717 & $0.419379-0.315329$ & $\mathrm{C}$ & $-0.615344 \quad 1.190130 \quad 0.406680$ \\
\hline $\mathrm{C}$ & 7.882615 & $1.773184-0.186306$ & $\mathrm{C}$ & $\begin{array}{lll}-1.528869 & 0.130752 & 0.401358\end{array}$ \\
\hline $\mathrm{C}$ & 8.083900 & $2.346266-1.480966$ & $\mathrm{C}$ & $-1.085248-1.196590 \quad 0.409504$ \\
\hline $\mathrm{N}$ & 8.296165 & $1.353825-2.398628$ & $\mathrm{C}$ & $0.290275-1.454786 \quad 0.420969$ \\
\hline $\mathrm{C}$ & 8.023271 & $3.742868-1.651464$ & $\mathrm{H}$ & $\begin{array}{lll}1.473452 & 1.729569 & 0.411960\end{array}$ \\
\hline $\mathrm{C}$ & 7.749114 & $4.527019-0.545087$ & $\mathrm{H}$ & $\begin{array}{lll}-2.598556 & 0.343560 & 0.447411\end{array}$ \\
\hline $\mathrm{C}$ & 7.524017 & $3.951830 \quad 0.730683$ & $\mathrm{C}$ & $-2.055062-2.318525 \quad 0.404039$ \\
\hline $\mathrm{C}$ & 7.584381 & $2.583406 \quad 0.927162$ & $\mathrm{H}$ & $0.646248-2.485827 \quad 0.381176$ \\
\hline $\mathrm{C}$ & 7.132408 & $-4.187565 \quad 3.120385$ & $\mathrm{C}$ & $-3.270587-2.236657-0.281829$ \\
\hline $\mathrm{H}$ & 7.299842 & -6.7616291 .509434 & $\mathrm{C}$ & $-4.148820-3.310538-0.270763$ \\
\hline $\mathrm{C}$ & 7.888671 & $-6.539265-1.371841$ & $\mathrm{~N}$ & $-3.878598-4.458333 \quad 0.377937$ \\
\hline $\mathrm{H}$ & 8.186781 & $4.178899-2.637845$ & $\mathrm{C}$ & $-2.716624-4.546317 \quad 1.055441$ \\
\hline $\mathrm{H}$ & 7.698566 & $5.611737-0.651261$ & $\mathrm{C}$ & $-1.795764-3.511491 \quad 1.088103$ \\
\hline $\mathrm{H}$ & 7.295276 & $\begin{array}{ll}4.606485 & 1.573309\end{array}$ & $\mathrm{H}$ & $-3.541454-1.345236-0.846630$ \\
\hline $\mathrm{H}$ & 7.390805 & $2.140775 \quad 1.906457$ & $\mathrm{H}$ & $-5.107204-3.258482-0.785991$ \\
\hline $\mathrm{H}$ & 7.969060 & $-4.539529 \quad 3.741494$ & $\mathrm{H}$ & $-2.566600-5.471374 \quad 1.610444$ \\
\hline $\mathrm{H}$ & 6.216870 & $-4.671387 \quad 3.489776$ & $\mathrm{H}$ & $-0.890635-3.633476 \quad 1.682197$ \\
\hline $\mathrm{H}$ & 7.045092 & $.099525 \quad 3.238736$ & & \\
\hline
\end{tabular}




\begin{tabular}{|c|c|c|c|c|}
\hline \multicolumn{3}{|c|}{ Complex 9' } & $\mathrm{C}$ & $1.753628-0.399379 \quad 1.102932$ \\
\hline $\mathrm{C}$ & $-3.329961 \quad 1.546353$ & 0.030423 & $\mathrm{H}$ & $1.163407-1.058211-2.035727$ \\
\hline $\mathrm{N}$ & $-2.999481 \quad 0.234841$ & 0.016359 & $\mathrm{H}$ & $3.644701-1.254648-2.084055$ \\
\hline $\mathrm{C}$ & $-3.933910-0.719710$ & 0.075543 & $\mathrm{C}$ & $5.348323-0.921876 \quad 0.056735$ \\
\hline $\mathrm{C}$ & $-5.290682-0.405654$ & 0.144397 & $\mathrm{H}$ & $3.631891-0.335296 \quad 2.133163$ \\
\hline $\mathrm{C}$ & $\begin{array}{ll}-5.638813 & 0.949442\end{array}$ & 0.144884 & $\mathrm{H}$ & $1.165747-0.132883 \quad 1.984281$ \\
\hline $\mathrm{C}$ & $-4.666713 \quad 1.940496$ & 0.089992 & $\mathrm{C}$ & $6.011470-1.885156-0.715132$ \\
\hline Ru & $-1.043719-0.238940$ & -0.039784 & $\mathrm{C}$ & $7.400074-1.982059-0.686994$ \\
\hline $\mathrm{N}$ & $-3.373408-2.021680$ & 0.072572 & $\mathrm{C}$ & $8.146868-1.114385 \quad 0.109074$ \\
\hline $\mathrm{H}$ & $-6.058990-1.167338$ & 0.203267 & $\mathrm{C}$ & $7.496673-0.150062 \quad 0.878250$ \\
\hline $\mathrm{H}$ & $-6.693442 \quad 1.220972$ & 0.196278 & $\mathrm{C}$ & $6.107854-0.055551 \quad 0.854539$ \\
\hline $\mathrm{H}$ & $-4.897825 \quad 3.004273$ & 0.095890 & $\mathrm{H}$ & $5.431227-2.581518-1.322878$ \\
\hline $\mathrm{N}$ & $-2.006131-2.123986$ & 0.096035 & $\mathrm{H}$ & $7.901282-2.743073-1.285997$ \\
\hline $\mathrm{C}$ & $-1.723250-3.416619$ & 0.088874 & $\mathrm{H}$ & $9.234588-1.188622 \quad 0.128735$ \\
\hline $\mathrm{C}$ & $-2.919344-4.174357$ & 0.061300 & $\mathrm{H}$ & $\begin{array}{lll}8.074552 & 0.538950 & 1.494998\end{array}$ \\
\hline $\mathrm{C}$ & $-3.953600-3.266564$ & 0.049035 & $\mathrm{H}$ & $\begin{array}{lll}5.606614 & 0.718473 & 1.438145\end{array}$ \\
\hline $\mathrm{C}$ & $\begin{array}{ll}-2.170893 & 2.433492\end{array}$ & -0.015512 & & \\
\hline $\mathrm{N}$ & $-0.929946 \quad 1.856008$ & -0.070106 & & plex 9'-ketone \\
\hline $\mathrm{C}$ & $-0.089249 \quad 2.930415$ & -0.114593 & $\mathrm{C}$ & $2.058228 \quad 0.303613-2.213598$ \\
\hline $\mathrm{C}$ & $-0.897920 \quad 4.107184$ & -0.071556 & $\mathrm{~N}$ & $1.947447-0.649452-1.256591$ \\
\hline $\mathrm{N}$ & $-2.221928 \quad 3.760429$ & -0.010310 & $\mathrm{C}$ & $2.209328-1.934719-1.537518$ \\
\hline $\mathrm{C}$ & $-0.295812 \quad 5.379658$ & -0.098229 & $\mathrm{C}$ & $2.609081-2.337585-2.810414$ \\
\hline $\mathrm{C}$ & $1.083458 \quad 5.447499$ & -0.172291 & $\mathrm{C}$ & $2.724849-1.355378-3.798499$ \\
\hline $\mathrm{C}$ & $1.879013 \quad 4.275159$ & -0.225367 & $\mathrm{C}$ & $2.447549-0.024832-3.510699$ \\
\hline $\mathrm{C}$ & $1.313811 \quad 3.012790$ & -0.199035 & $\mathrm{Ru}$ & $1.436469-0.104365 \quad 0.571414$ \\
\hline $\mathrm{C}$ & $-0.304252-3.878511$ & 0.072120 & $\mathrm{~N}$ & $2.036674-2.778007-0.413137$ \\
\hline $\mathrm{H}$ & $-3.016926-5.255163$ & 0.048612 & $\mathrm{H}$ & $2.828861-3.372729-3.042616$ \\
\hline $\mathrm{C}$ & $-5.420240-3.537956$ & 0.010994 & $\mathrm{H}$ & $3.036360-1.647886-4.801202$ \\
\hline $\mathrm{H}$ & $-0.914605 \quad 6.277111$ & -0.063989 & $\mathrm{H}$ & $2.518748 \quad 0.773081-4.247991$ \\
\hline $\mathrm{H}$ & $1.575732 \quad 6.420829$ & -0.196369 & $\mathrm{~N}$ & $1.660727-2.190201 \quad 0.766102$ \\
\hline $\mathrm{H}$ & $2.963476 \quad 4.376136$ & $5-0.293945$ & $\mathrm{C}$ & $1.567373-3.160646 \quad 1.661741$ \\
\hline $\mathrm{H}$ & $1.927980 \quad 2.111511$ & -0.252086 & $\mathrm{C}$ & $1.883677-4.404417 \quad 1.060709$ \\
\hline $\mathrm{H}$ & $-0.223754-4.900210$ & 0.463800 & $\mathrm{C}$ & $2.179703-4.135857-0.255178$ \\
\hline $\mathrm{H}$ & $0.320487-3.206663$ & 0.675575 & $\mathrm{C}$ & $1.707191 \quad 1.639999-1.742297$ \\
\hline $\mathrm{H}$ & $0.090939-3.870107$ & -0.955068 & $\mathrm{~N}$ & $1.361542 \quad 1.776637-0.426919$ \\
\hline $\mathrm{H}$ & $-5.899119-3.066704$ & -0.858796 & $\mathrm{C}$ & $0.995353 \quad 3.087941-0.338328$ \\
\hline $\mathrm{H}$ & $-5.926879-3.186467$ & 0.921183 & $\mathrm{C}$ & $1.172295 \quad 3.672009-1.631695$ \\
\hline $\mathrm{H}$ & $-5.568472-4.621509$ & -0.062466 & $\mathrm{~N}$ & $1.622143 \quad 2.724387-2.509984$ \\
\hline $\mathrm{H}$ & $-1.100694-0.415521$ & -1.556970 & $\mathrm{C}$ & $0.867797 \quad 5.031198-1.839890$ \\
\hline $\mathrm{N}$ & $1.053052-0.602601$ & -0.027885 & $\mathrm{C}$ & $0.384032 \quad 5.765679-0.772110$ \\
\hline $\mathrm{C}$ & $1.754960-0.916505$ & -1.131719 & $\mathrm{C}$ & $\begin{array}{lll}0.186530 & 5.175300 & 0.501305\end{array}$ \\
\hline $\mathrm{C}$ & $3.138736-1.030894$ & -1.144817 & $\mathrm{C}$ & $\begin{array}{llll}0.483163 & 3.844156 & 0.734651\end{array}$ \\
\hline $\mathrm{C}$ & $3.870574-0.818764$ & 0.029222 & $\mathrm{C}$ & $1.199865-2.856670 \quad 3.076219$ \\
\hline $\mathrm{C}$ & $3.135297-0.497153$ & 1.176640 & $\mathrm{H}$ & $\begin{array}{lll}1.897980 & -5.382345 & 1.531219\end{array}$ \\
\hline
\end{tabular}




\begin{tabular}{|c|c|c|c|}
\hline $\mathrm{C}$ & $2.576879-5.097657-1.324403$ & $\mathrm{Col}$ & aplex 9'-ketone (TS) \\
\hline $\mathrm{H}$ & $1.009256 \quad 5.476637-2.825423$ & $\mathrm{C}$ & $\begin{array}{lll}-1.793509 & 0.772341 & 2.153970\end{array}$ \\
\hline $\mathrm{H}$ & $0.139022 \quad 6.820004-0.908679$ & $\mathrm{~N}$ & $-1.769060-0.300357 \quad 1.331796$ \\
\hline & $\begin{array}{lll}-0.214908 & 5.786909 & 1.311076\end{array}$ & $\mathrm{C}$ & $-2.091957-1.524594 \quad 1.764428$ \\
\hline & $\begin{array}{lll}0.308227 & 3.385633 & 1.710128\end{array}$ & $\mathrm{C}$ & $-2.492569-1.740993 \quad 3.081665$ \\
\hline & $2.050481-3.050456 \quad 3.746020$ & $\mathrm{C}$ & $-2.515949-0.635707 \quad 3.940836$ \\
\hline & $0.362392-3.486445 \quad 3.409304$ & $\mathrm{C}$ & $\begin{array}{lll}-2.160007 & 0.629439 & 3.493848\end{array}$ \\
\hline & $0.919538-1.798862 \quad 3.165682$ & $\mathrm{Ru}$ & $-1.360789-0.000828-0.617706$ \\
\hline H & $3.581497-4.882752-1.715425$ & $\mathrm{~N}$ & $-1.989158-2.512847 \quad 0.751322$ \\
\hline $\mathrm{H}$ & $1.867790-5.092769-2.164330$ & $\mathrm{H}$ & $-2.788367-2.718610 \quad 3.442723$ \\
\hline $\mathrm{H}$ & $2.588285-6.105616-0.893649$ & $\mathrm{H}$ & $-2.823987-0.782478 \quad 4.976275$ \\
\hline I & $\begin{array}{lll}2.965891 & 0.061330 & 0.889746\end{array}$ & $\mathrm{H}$ & $-2.159914 \quad 1.510648 \quad 4.133058$ \\
\hline $\mathrm{N}$ & $-0.863627-0.282962 \quad 0.219066$ & $\mathrm{~N}$ & $-1.682074-2.104940-0.522828$ \\
\hline $\mathrm{C}$ & $-1.581549-1.180738 \quad 0.906139$ & $\mathrm{C}$ & $-1.558314-3.198114-1.257789$ \\
\hline$C$ & $-2.960128-1.309865 \quad 0.790850$ & $\mathrm{C}$ & $-1.794818-4.343724-0.456255$ \\
\hline$c$ & $-3.658215-0.470190-0.085006$ & $\mathrm{C}$ & $-2.059712-3.884777 \quad 0.812166$ \\
\hline $\mathrm{C}$ & 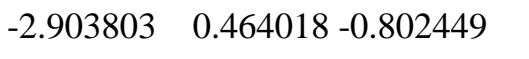 & $\mathrm{C}$ & $\begin{array}{lll}-1.369825 & 2.004817 & 1.498597\end{array}$ \\
\hline C & $-1.526220 \quad 0.524138-0.621241$ & $\mathrm{~N}$ & $\begin{array}{lll}-1.090568 & 1.948527 & 0.159484\end{array}$ \\
\hline $\mathrm{H}$ & $-1.013105-1.820346 \quad 1.585306$ & $\mathrm{C}$ & $-0.580278 \quad 3.183785-0.105055$ \\
\hline $\mathrm{H}$ & $-3.487655-2.043831 \quad 1.400439$ & $\mathrm{C}$ & $\begin{array}{lll}-0.613506 & 3.929995 & 1.112835\end{array}$ \\
\hline $\mathrm{C}$ & $-5.129732-0.565895-0.243036$ & $\mathrm{~N}$ & $\begin{array}{lll}-1.115307 & 3.153343 \quad 2.121693\end{array}$ \\
\hline $\mathrm{H}$ & $-3.378378 \quad 1.134236-1.519279$ & $\mathrm{C}$ & $-0.142269 \quad 5.258486 \quad 1.136856$ \\
\hline $\mathrm{H}$ & $-0.918693 \quad 1.249470-1.166761$ & $\mathrm{C}$ & $0.355473 \quad 5.793542-0.035966$ \\
\hline $\mathrm{C}$ & $-5.772563-1.810314-0.206612$ & $\mathrm{C}$ & $0.402613 \quad 5.037164-1.236498$ \\
\hline $\mathrm{C}$ & $-7.153883-1.899510-0.357628$ & $\mathrm{C}$ & $-0.057455 \quad 3.735032-1.292151$ \\
\hline C & $-7.914285-0.745419-0.542145$ & $\mathrm{C}$ & $-1.202874-3.109288-2.706592$ \\
\hline $\mathrm{C}$ & 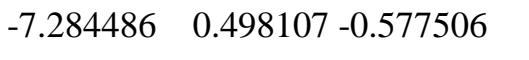 & $\mathrm{H}$ & $-1.769787-5.383272-0.766878$ \\
\hline $\mathrm{C}$ & $\begin{array}{llll}-5.902626 & 0.587481 & -0.431688\end{array}$ & $\mathrm{C}$ & $-2.352252-4.684716 \quad 2.037247$ \\
\hline $\mathrm{H}$ & $-5.179724-2.718622-0.085926$ & $\mathrm{H}$ & $\begin{array}{lll}-0.171553 & 5.832345 & 2.063859\end{array}$ \\
\hline $\mathrm{H}$ & $-7.638237-2.876437-0.337859$ & $\mathrm{H}$ & $0.728309 \quad 6.818867-0.044952$ \\
\hline $\mathrm{H}$ & $-8.996266-0.815063-0.658926$ & $\mathrm{H}$ & $0.816905 \quad 5.498650-2.134252$ \\
\hline $\mathrm{H}$ & $\begin{array}{llll}-7.873099 & 1.405931 & -0.713918\end{array}$ & $\mathrm{H}$ & $-0.016072 \quad 3.139975-2.206333$ \\
\hline $\mathrm{H}$ & 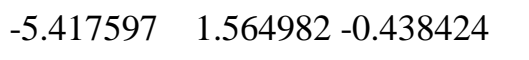 & $\mathrm{H}$ & $-1.974182-3.591854-3.324448$ \\
\hline $\mathrm{O}$ & $\begin{array}{lll}0.872153 & 0.550122 & 2.587247\end{array}$ & $\mathrm{H}$ & $-0.253227-3.630810-2.897891$ \\
\hline $\mathrm{C}$ & $\begin{array}{lll}1.422849 & 1.393247 & 3.287446\end{array}$ & $\mathrm{H}$ & $-1.115735-2.053769-3.004673$ \\
\hline $\mathrm{C}$ & $\begin{array}{lll}0.828750 & 1.747750 & 4.621693\end{array}$ & $\mathrm{H}$ & $-3.367389-4.499178 \quad 2.415835$ \\
\hline $\mathrm{C}$ & $\begin{array}{lll}2.683388 & 2.085595 & 2.864399\end{array}$ & $\mathrm{H}$ & $-1.634992-4.472805 \quad 2.842511$ \\
\hline $\mathrm{H}$ & $\begin{array}{lll}0.518330 & 2.804304 & 4.602105\end{array}$ & $\mathrm{H}$ & $-2.275445-5.747521 \quad 1.780647$ \\
\hline $\mathrm{H}$ & $\begin{array}{lll}1.592874 & 1.654811 & 5.407910\end{array}$ & $\mathrm{H}$ & $-2.926652 \quad 0.318344-0.974607$ \\
\hline $\mathrm{H}$ & $\begin{array}{lll}-0.033552 & 1.109597 & 4.842245\end{array}$ & $\mathrm{~N}$ & $0.783393-0.315357-0.275002$ \\
\hline $\mathrm{H}$ & $2.875738 \quad 2.980692 \quad 3.469086$ & $\mathrm{C}$ & $1.480172-0.991318-1.201236$ \\
\hline 11 & $\begin{array}{lll}2.638756 & 2.321254 & 1.792572\end{array}$ & $\mathrm{C}$ & $2.857981-1.149173-1.145498$ \\
\hline & $3.512652 \quad 1.370321 \quad 2.988351$ & $\mathrm{C}$ & $3.576838-0.584382-0.085215$ \\
\hline & & $\mathrm{C}$ & $\begin{array}{lll}2.842701 & 0.124068 & 0.871761\end{array}$ \\
\hline
\end{tabular}


$\mathrm{H}$

$\mathrm{H}$

C

$\mathrm{H}$

$\mathrm{H}$

\section{C}

C

C

C

C

C

$\mathrm{H}$

\section{H}

$\mathrm{H}$

$\mathrm{H}$

$\mathrm{H}$

O

\section{C}

C

$\mathrm{C}$

\section{Complex 10' \\ $\begin{array}{llll}\text { C } & -5.490304 & 1.233195 & -0.288928\end{array}$ \\ $\begin{array}{llll}\mathrm{N} & -5.021638 & 0.002496 & 0.018603\end{array}$ \\ $\begin{array}{llll}\text { C } & -5.847810 & -0.994571 & 0.351184\end{array}$ \\ $\begin{array}{llll}\text { C } & -7.229791 & -0.812869 & 0.384704\end{array}$ \\ $\begin{array}{lllll}\text { C } & -7.720965 & 0.454547 & 0.052974\end{array}$ \\ $\begin{array}{llll}\text { C } & -6.861018 & 1.491986 & -0.285017\end{array}$ \\ $\begin{array}{llll}\mathrm{Ru} & -3.024923 & -0.266540 & 0.020672\end{array}$ \\ $\begin{array}{lll}\mathrm{N} & -5.151395-2.189449 & 0.662022\end{array}$ \\ $\mathrm{H} \quad-7.911769-1.607824 \quad 0.661796$ \\ $\begin{array}{lllll}\mathrm{H} & -8.798204 & 0.621430 & 0.070041\end{array}$ \\ $\begin{array}{llll}\mathrm{H} & -7.204411 & 2.492685 & -0.541378\end{array}$ \\ $\begin{array}{llll}\mathrm{N} & -3.780937-2.142502 & 0.667911\end{array}$ \\ C $\quad-3.362495-3.357483 \quad 0.984011$ \\ $\begin{array}{lll}\text { C } & -4.471211-4.214117 & 1.189472\end{array}$ \\ C $\quad-5.596062-3.450357 \quad 0.977181$ \\ C $\quad-4.433457 \quad 2.191968-0.599250$}

$\begin{array}{llll}\mathrm{N} & -3.138031 & 1.748771 & -0.550886\end{array}$

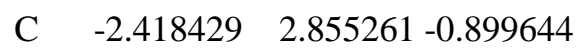

C $\quad-3.348533 \quad 3.915318-1.126480$

N $\quad-4.626828 \quad 3.463214-0.929725$

C $\quad-2.8878625 .192438-1.499121$

C $\quad-1.524941 \quad 5.378847-1.641556$

C $\quad-0.608362 \quad 4.318844-1.427169$

C $\quad-1.033787 \quad 3.055012-1.058973$

C $\quad-1.902970-3.663021 \quad 1.043315$

H $\quad-4.453266-5.265555 \quad 1.457720$

C $\quad-7.025546-3.870394 \quad 1.056310$

H $\quad-3.599664 \quad 6.000767-1.670495$

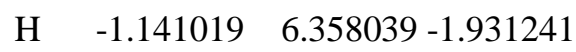

$\mathrm{H} \quad 0.457832 \quad 4.507778-1.563244$

$\begin{array}{llll}\mathrm{H} & -0.326403 & 2.236883 & -0.909484\end{array}$

$\begin{array}{llll}\mathrm{H} & -1.710760 & -4.512489 & 1.710707\end{array}$

$\begin{array}{llll}\mathrm{H} & -1.347010 & -2.786125 & 1.400534\end{array}$

$\mathrm{H} \quad-1.521929-3.918558 \quad 0.042847$

$\mathrm{H} \quad-7.555904-3.689840 \quad 0.110820$

$\begin{array}{llll}\mathrm{H} & -7.562398 & -3.350314 & 1.862480\end{array}$

H $\quad-7.057692-4.945659 \quad 1.266511$

H $\quad-3.066015-0.833265-1.397711$

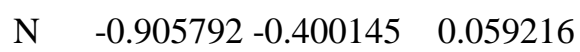

C $\quad-0.182812-0.980029-0.916246$

C $\quad 1.205387-0.971343-0.939549$

$\begin{array}{llll}\text { C } & 1.917923-0.339755 & 0.085681\end{array}$

$\begin{array}{llll}\text { C } & 1.162801 & 0.260642 & 1.099623\end{array}$

$\begin{array}{llll}\text { C } & -0.222518 & 0.208961 & 1.045989\end{array}$

H $\quad-0.762845-1.449611-1.710099$

H $\quad 1.725969-1.432663-1.778892$

C $\quad 3.399749-0.304560 \quad 0.095205$

$\begin{array}{llll}\mathrm{H} & 1.646481 & 0.757492 & 1.940499\end{array}$

$\begin{array}{llll}\mathrm{H} & -0.829028 & 0.687418 & 1.818198\end{array}$

C $\quad 4.136880-1.410705-0.340628$

C $5.535101-1.392316-0.330091$

$\begin{array}{llll}\text { C } & 6.194304 & -0.241796 & 0.114019\end{array}$

$\begin{array}{llll}\text { C } & 5.478045 & 0.878997 & 0.546615\end{array}$

$\begin{array}{lllll}\text { C } & 4.080130 & 0.834735 & 0.538631\end{array}$

H $\quad 3.614910-2.319832-0.644977$

C $\quad 6.307198-2.580730-0.771693$

$\mathrm{H} \quad 7.285508-0.217648 \quad 0.121511$

$\begin{array}{lllll}\text { C } & 6.192245 & 2.101777 & 0.992486\end{array}$

$\begin{array}{llll}\mathrm{H} & 3.513265 & 1.720703 & 0.830916\end{array}$

$\begin{array}{llll}\text { C } & 7.361971 & 2.534481 & 0.359729\end{array}$ 


$\begin{array}{llll}\mathrm{C} & 7.994011 & 3.687816 & 0.819239 \\ \mathrm{~N} & 7.554904 & 4.424901 & 1.842441 \\ \mathrm{C} & 6.436841 & 4.007716 & 2.441656 \\ \mathrm{C} & 5.725770 & 2.871134 & 2.063009 \\ \mathrm{C} & 7.480407 & -2.973945 & -0.119988 \\ \mathrm{C} & 8.164818 & -4.098259 & -0.576531 \\ \mathrm{~N} & 7.774100 & -4.842489 & -1.614257 \\ \mathrm{C} & 6.654002 & -4.461220 & -2.233386 \\ \mathrm{C} & 5.892688 & -3.356286 & -1.859083 \\ \mathrm{H} & 7.764757 & 1.996845 & -0.499468 \\ \mathrm{H} & 8.905370 & 4.041696 & 0.329971 \\ \mathrm{H} & 6.087267 & 4.615210 & 3.280655 \\ \mathrm{H} & 4.831779 & 2.578010 & 2.614783 \\ \mathrm{H} & 7.846016 & -2.427807 & 0.750449 \\ \mathrm{H} & 9.079594 & -4.421461 & -0.072555 \\ \mathrm{H} & 6.346183 & -5.072090 & -3.086234 \\ \mathrm{H} & 5.000916 & -3.089553 & -2.427673\end{array}$

\section{Complex 10'-ketone}

$\begin{array}{cccc}\mathrm{C} & 3.773907 & 0.184900 & -2.277666 \\ \mathrm{~N} & 3.638455 & -0.765524 & -1.321478 \\ \mathrm{C} & 3.798128 & -2.063575 & -1.619388 \\ \mathrm{C} & 4.111308 & -2.483598 & -2.910813 \\ \mathrm{C} & 4.251619 & -1.504171 & -3.898457 \\ \mathrm{C} & 4.081803 & -0.159738 & -3.592341 \\ \mathrm{Ru} & 3.245573 & -0.199909 & 0.530369 \\ \mathrm{~N} & 3.617901 & -2.901351 & -0.491901 \\ \mathrm{H} & 4.246658 & -3.529769 & -3.157642 \\ \mathrm{H} & 4.496853 & -1.809928 & -4.915496 \\ \mathrm{H} & 4.176810 & 0.636884 & -4.328355 \\ \mathrm{~N} & 3.331074 & -2.297980 & 0.704370 \\ \mathrm{C} & 3.213783 & -3.266024 & 1.600031 \\ \mathrm{C} & 3.423583 & -4.523735 & 0.981698 \\ \mathrm{C} & 3.680388 & -4.266497 & -0.344757 \\ \mathrm{C} & 3.540045 & 1.539191 & -1.784663 \\ \mathrm{~N} & 3.265290 & 1.690031 & -0.454045 \\ \mathrm{C} & 3.000945 & 3.024071 & -0.342451 \\ \mathrm{C} & 3.160900 & 3.603940 & -1.639836 \\ \mathrm{~N} & 3.500167 & 2.632997 & -2.542168 \\ \mathrm{C} & 2.946076 & 4.983125 & -1.827522 \\ \mathrm{C} & 2.564376 & 5.741681 & -0.735481 \\ \mathrm{C} & 2.381547 & 5.156794 & 0.542659 \\ \mathrm{C} & 2.592100 & 3.805996 & 0.756116 \\ \mathrm{C} & 2.927703 & -2.946680 & 3.029861\end{array}$

$\mathrm{H} \quad 3.075239 \quad 5.425249-2.816210$

$\mathrm{H} \quad 2.390276 \quad 6.811935-0.855751$

$\begin{array}{llll}\mathrm{H} & 2.061506 & 5.789141 & 1.372439\end{array}$

$\mathrm{H} \quad 2.428611 \quad 3.353054 \quad 1.736222$

H $\quad 3.799761-3.178966 \quad 3.658652$

H $\quad 2.079851-3.538537 \quad 3.403490$

H $\quad 2.700835-1.877193 \quad 3.131408$

H $\quad 4.968325-5.088663-1.867621$

H $\quad 3.226865-5.193034-2.240506$

H $\quad 3.940495-6.254013-1.005814$

H $\quad 4.794038-0.146423 \quad 0.780841$

N $\quad 0.919690-0.206460 \quad 0.279993$

C $\quad 0.173764-1.099452 \quad 0.942671$

C $\quad-1.215153-1.119419 \quad 0.899994$

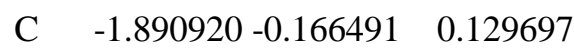

C $\quad-1.108474 \quad 0.761766-0.563686$

$\begin{array}{llll}\text { C } & 0.277657 & 0.707059 & -0.461377\end{array}$

H $\quad 0.727328-1.828402 \quad 1.539101$

H $\quad-1.764032-1.857485 \quad 1.485390$

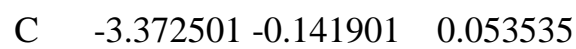

$\mathrm{H} \quad-1.567864 \quad 1.516772-1.201838$

$\begin{array}{llll}\mathrm{H} & 0.909273 & 1.424113 & -0.989777\end{array}$

C $\quad-4.101661-1.335137 \quad 0.024288$

C $\quad-5.497993-1.323195-0.054384$

C $\quad-6.164318-0.094286-0.092575$

C $\quad-5.456363 \quad 1.111369-0.058046$

$\begin{array}{llll}\text { C } & -4.059769 & 1.075684 & 0.009393\end{array}$

H $\quad-3.571266-2.289059 \quad 0.009908$

C $\quad-6.260672-2.595469-0.110214$

H $\quad-7.254144-0.075802-0.150272$

$\begin{array}{llll}\text { C } & -6.177088 & 2.409031 & -0.080219\end{array}$

$\begin{array}{llll}\mathrm{H} & -3.500850 & 2.010713 & 0.078908\end{array}$

$\begin{array}{llll}\text { O } & 2.817814 & 0.477107 & 2.572656\end{array}$

C $\quad 3.460284 \quad 1.269816 \quad 3.253542$

$\begin{array}{llll}\text { C } & 2.954242 & 1.652869 & 4.615808\end{array}$

$\begin{array}{llll}\text { C } & 4.748476 & 1.871893 & 2.779090\end{array}$

$\mathrm{H} \quad 2.719536 \quad 2.728988 \quad 4.617211$

$\begin{array}{llll}\mathrm{H} & 3.744613 & 1.498462 & 5.365498\end{array}$

$\begin{array}{llll}\mathrm{H} & 2.059457 & 1.075653 & 4.871903\end{array}$

$\begin{array}{llll}\mathrm{H} & 5.032022 & 2.745203 & 3.379705\end{array}$

$\begin{array}{llll}\mathrm{H} & 4.674844 & 2.119534 & 1.711598\end{array}$

$\begin{array}{llll}\mathrm{H} & 5.527841 & 1.097106 & 2.863039\end{array}$ 


$\begin{array}{llll}\mathrm{C} & -5.674677 & 3.510792 & -0.779789 \\ \mathrm{C} & -6.392155 & 4.704882 & -0.769864 \\ \mathrm{~N} & -7.549319 & 4.877492 & -0.125906 \\ \mathrm{C} & -8.023166 & 3.822482 & 0.541560 \\ \mathrm{C} & -7.388429 & 2.583615 & 0.596798 \\ \mathrm{C} & -5.876050 & -3.710587 & 0.641346 \\ \mathrm{C} & -6.627899 & -4.880022 & 0.553708 \\ \mathrm{~N} & -7.712100 & -5.017275 & -0.213644 \\ \mathrm{C} & -8.073981 & -3.949707 & -0.929687 \\ \mathrm{C} & -7.395817 & -2.732864 & -0.915598 \\ \mathrm{H} & -4.746989 & 3.436705 & -1.348550 \\ \mathrm{H} & -6.014245 & 5.572097 & -1.317777 \\ \mathrm{H} & -8.967224 & 3.975200 & 1.071452 \\ \mathrm{H} & -7.822544 & 1.773351 & 1.183858 \\ \mathrm{H} & -5.014869 & -3.663453 & 1.309037 \\ \mathrm{H} & -6.342685 & -5.756976 & 1.141094 \\ \mathrm{H} & -8.958733 & -4.073063 & -1.559953 \\ \mathrm{H} & -7.735536 & -1.910995 & -1.547008\end{array}$

\section{Complex 10'-ketone (TS)}

$\begin{array}{llll}\text { C } & -3.615220 & 1.014346 & 2.015818\end{array}$

$\begin{array}{llll}\mathrm{N} & -3.504423 & -0.185871 & 1.403481\end{array}$

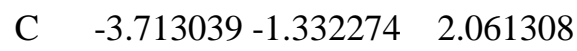

C $\quad-4.077334-1.334861 \quad 3.406555$

C $\quad-4.189159-0.096066 \quad 4.049238$

$\begin{array}{llll}\text { C } & -3.952518 & 1.090558 & 3.368621\end{array}$

Ru $\quad-3.144454-0.217795-0.577778$

$\begin{array}{llll}\mathrm{N} & -3.534803 & -2.478360 & 1.243563\end{array}$

$\mathrm{H} \quad-4.280419-2.250451 \quad 3.948752$

H $\quad-4.470917-0.074833 \quad 5.102188$

$\mathrm{H} \quad-4.024018 \quad 2.071203 \quad 3.835854$

N $\quad-3.274678-2.288039-0.090732$

C $\quad-3.067012-3.484136-0.617049$

C $\quad-3.199753-4.478311 \quad 0.385228$

C $\quad-3.490407-3.817083 \quad 1.554920$

$\begin{array}{llll}\text { C } & -3.309714 & 2.135846 & 1.133905\end{array}$

N $\quad-3.046844 \quad 1.856323-0.180408$

$\begin{array}{llll}\text { C } & -2.644837 & 3.057929 & -0.681244\end{array}$

$\begin{array}{llll}\text { C } & -2.721750 & 4.010810 & 0.380329\end{array}$

$\begin{array}{llll}\mathrm{N} & -3.141861 & 3.395972 & 1.528798\end{array}$

$\begin{array}{llll}\text { C } & -2.361803 & 5.353800 & 0.147027\end{array}$

C $\quad-1.926514 \quad 5.700171-1.117957$

C $\quad-1.833899 \quad 4.741149-2.160718$

C $\quad-2.185999 \quad 3.419246-1.963913$
C $\quad-2.733857-3.634321-2.066127$

H $\quad-3.091568-5.551839 \quad 0.268493$

$\begin{array}{llll}\text { C } & -3.705598 & -4.400021 & 2.911495\end{array}$

$\begin{array}{llll}\mathrm{H} & -2.425537 & 6.085198 & 0.953539\end{array}$

H $\quad-1.640335 \quad 6.732312-1.325456$

$\mathrm{H} \quad-1.472078 \quad 5.060184-3.139397$

H $\quad-2.109342 \quad 2.670453-2.754509$

H $\quad-3.465989-4.285087-2.566067$

H $\quad-1.744609-4.101540-2.182795$

H $\quad-2.741648-2.648351-2.554374$

H $\quad-4.728439-4.226887 \quad 3.274785$

H $\quad-2.999327-3.990637 \quad 3.647291$

H $\quad-3.546808-5.4827112 .849825$

H $\quad-4.738702-0.110707-0.957164$

$\mathrm{N} \quad-0.978109-0.263314-0.250373$

C $\quad-0.235410-1.041821-1.051908$

C $\quad 1.152400-1.049563-1.024285$

C $\quad 1.829308-0.213151-0.129620$

$\begin{array}{llll}\text { C } & 1.047815 & 0.598684 & 0.698151\end{array}$

$\begin{array}{llll}\text { C } & -0.337250 & 0.546445 & 0.605252\end{array}$

H $\quad-0.795052-1.657849-1.757276$

H $\quad 1.699608-1.681321-1.723823$

C $\quad 3.310936-0.183429-0.066740$

$\begin{array}{llll}\mathrm{H} & 1.508473 & 1.263574 & 1.428466\end{array}$

$\begin{array}{llll}\mathrm{H} & -0.964594 & 1.183684 & 1.231507\end{array}$

C $\quad 4.049944-1.361429-0.216203$

C $\quad 5.446691-1.346597-0.147929$

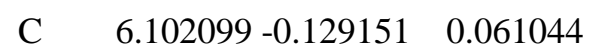

$\begin{array}{llll}\text { C } & 5.383784 & 1.062591 & 0.203614\end{array}$

$\begin{array}{llll}\text { C } & 3.986947 & 1.023046 & 0.143713\end{array}$

H $\quad 3.528582-2.312953-0.336310$

C $\quad 6.220754-2.606005-0.282784$

H $\quad 7.192230-0.108586 \quad 0.111975$

$\begin{array}{llll}\text { C } & 6.094331 & 2.350894 & 0.402346\end{array}$

$\begin{array}{llll}\mathrm{H} & 3.419363 & 1.953139 & 0.209431\end{array}$

O $\quad-3.153287-0.348687-2.698320$

C $\quad-4.422632-0.164126-2.422611$

C $\quad-5.357698-1.334027-2.722869$

C $\quad-5.012997 \quad 1.186465-2.803888$

H $\quad-5.380630-1.474247-3.815139$

H $\quad-6.379532-1.134235-2.371496$

$\mathrm{H} \quad-4.995222-2.256978-2.255568$

$\mathrm{H} \quad-6.004280 \quad 1.338657-2.354974$

$\begin{array}{llll}\mathrm{H} & -5.116303 & 1.205297 & -3.900748\end{array}$ 


$\begin{array}{cccc}\mathrm{H} & -4.348971 & 2.000676 & -2.496317 \\ \mathrm{C} & 5.588670 & 3.339874 & 1.251940 \\ \mathrm{C} & 6.297355 & 4.529679 & 1.404576 \\ \mathrm{~N} & 7.448476 & 4.799804 & 0.784179 \\ \mathrm{C} & 7.924847 & 3.852848 & -0.027773 \\ \mathrm{C} & 7.298957 & 2.628622 & -0.251723 \\ \mathrm{C} & 5.844772 & -3.598614 & -1.193479 \\ \mathrm{C} & 6.606399 & -4.761601 & -1.281795 \\ \mathrm{~N} & 7.691686 & -5.003381 & -0.542203 \\ \mathrm{C} & 8.045534 & -4.052317 & 0.325901 \\ \mathrm{C} & 7.357489 & -2.852412 & 0.493578 \\ \mathrm{H} & 4.665030 & 3.180192 & 1.809504 \\ \mathrm{H} & 5.916667 & 5.309164 & 2.069823 \\ \mathrm{H} & 8.863331 & 4.086437 & -0.537621 \\ \mathrm{H} & 7.734319 & 1.912782 & -0.950014 \\ \mathrm{H} & 4.983495 & -3.458344 & -1.847871 \\ \mathrm{H} & 6.328895 & -5.542501 & -1.994919 \\ \mathrm{H} & 8.931619 & -4.261695 & 0.931100 \\ \mathrm{H} & 7.691072 & -2.130776 & 1.240312\end{array}$

\section{Complex 10'- $\mathbf{P P h}_{3}$}

$\begin{array}{lrrr}\mathrm{P} & 1.059505 & -1.129718 & -1.180046 \\ \mathrm{C} & 2.273217 & -1.040861 & -2.583546 \\ \mathrm{C} & -0.585589 & -1.329842 & -2.020590 \\ \mathrm{C} & 1.427176 & -2.854682 & -0.622502 \\ \mathrm{C} & 2.211341 & -1.920749 & -3.672643 \\ \mathrm{C} & 3.166025 & -1.863664 & -4.685122 \\ \mathrm{C} & 4.208698 & -0.938424 & -4.611519 \\ \mathrm{C} & 4.293287 & -0.078214 & -3.518970 \\ \mathrm{C} & 3.330333 & -0.128927 & -2.509295 \\ \mathrm{C} & 0.801619 & -3.983159 & -1.164843 \\ \mathrm{C} & 1.107269 & -5.254958 & -0.680635 \\ \mathrm{C} & 2.046854 & -5.408640 & 0.338062 \\ \mathrm{C} & 2.684803 & -4.288155 & 0.868297 \\ \mathrm{C} & 2.375355 & -3.015357 & 0.394454 \\ \mathrm{C} & -0.859077 & -0.953867 & -3.340246 \\ \mathrm{C} & -2.168809 & -0.969215 & -3.829506 \\ \mathrm{C} & -3.221067 & -1.368962 & -3.010847 \\ \mathrm{C} & -2.958801 & -1.754859 & -1.694384 \\ \mathrm{C} & -1.657788 & -1.726602 & -1.203495 \\ \mathrm{H} & 1.410586 & -2.661780 & -3.728411 \\ \mathrm{H} & 3.102263 & -2.551141 & -5.529732 \\ \mathrm{H} & 4.960203 & -0.898391 & -5.401383 \\ \mathrm{H} & 5.115364 & 0.635594 & -3.446562\end{array}$

\begin{tabular}{|c|c|c|c|}
\hline $\mathrm{H}$ & 3.391107 & 0.538374 & -1.647245 \\
\hline $\mathrm{H}$ & 0.051012 & -3.871363 & -1.950046 \\
\hline $\mathrm{H}$ & 0.603892 & -6.128201 & -1.098317 \\
\hline $\mathrm{H}$ & 2.275946 & -6.402758 & 0.724313 \\
\hline $\mathrm{H}$ & 3.409775 & -4.401535 & 1.675030 \\
\hline $\mathrm{H}$ & 2.835155 & -2.130784 & 0.839124 \\
\hline $\mathrm{H}$ & -0.047765 & -0.644864 & -4.001685 \\
\hline $\mathrm{H}$ & -2.360745 & -0.669822 & -4.861103 \\
\hline $\mathrm{H}$ & -4.243196 & -1.378630 & -3.392043 \\
\hline $\mathrm{H}$ & -3.776104 & -2.064775 & -1.040828 \\
\hline $\mathrm{H}$ & -1.471694 & -1.997789 & -0.161448 \\
\hline $\mathrm{C}$ & 1.598272 & 1.491368 & 3.137929 \\
\hline $\mathrm{N}$ & 1.626201 & 1.895505 & 1.856159 \\
\hline $\mathrm{C}$ & 2.104998 & 3.092466 & 1.521380 \\
\hline $\mathrm{C}$ & 2.596237 & 3.977383 & 2.480449 \\
\hline $\mathrm{C}$ & 2.545963 & 3.570331 & 3.818208 \\
\hline $\mathrm{C}$ & 2.042129 & 2.326331 & 4.166327 \\
\hline $\mathrm{Ru}$ & 1.156689 & 0.491641 & 0.388712 \\
\hline $\mathrm{N}$ & 2.057713 & 3.333285 & 0.125223 \\
\hline $\mathrm{H}$ & 3.015134 & 4.941686 & 2.222590 \\
\hline $\mathrm{H}$ & 2.920177 & 4.245704 & 4.588041 \\
\hline $\mathrm{H}$ & 1.988586 & 1.964308 & 5.191369 \\
\hline $\mathrm{N}$ & 1.605487 & 2.329016 & -0.696797 \\
\hline $\mathrm{C}$ & 1.556209 & 2.849852 & -1.917690 \\
\hline $\mathrm{C}$ & 1.988406 & 4.196817 & -1.894087 \\
\hline $\mathrm{C}$ & 2.295456 & 4.483071 & -0.586866 \\
\hline $\mathrm{C}$ & 1.075164 & 0.145306 & 3.324586 \\
\hline $\mathrm{N}$ & 0.788190 & -0.621102 & 2.225778 \\
\hline $\mathrm{C}$ & 0.255532 & -1.755589 & 2.787711 \\
\hline $\mathrm{C}$ & 0.275242 & -1.584232 & 4.207653 \\
\hline $\mathrm{N}$ & 0.805069 & -0.365307 & 4.520982 \\
\hline $\mathrm{C}$ & -0.225229 & -2.582112 & 5.066826 \\
\hline $\mathrm{C}$ & -0.747818 & -3.729001 & 4.502460 \\
\hline $\mathrm{C}$ & -0.778852 & -3.897433 & 3.096332 \\
\hline $\mathrm{C}$ & -0.288300 & -2.934460 & 2.233058 \\
\hline $\mathrm{C}$ & 1.065661 & 2.091519 & -3.104671 \\
\hline $\mathrm{H}$ & 2.056405 & 4.879937 & -2.734569 \\
\hline $\mathrm{C}$ & 2.771625 & 5.782758 & -0.028655 \\
\hline $\mathrm{H}$ & -0.195275 & -2.429964 & 6.146424 \\
\hline $\mathrm{H}$ & -1.142686 & -4.519483 & 5.142427 \\
\hline $\mathrm{H}$ & -1.192090 & -4.817592 & 2.679828 \\
\hline $\mathrm{H}$ & -0.297976 & -3.109966 & 1.159777 \\
\hline $\mathrm{H}$ & 1.889596 & 1.845950 & -3.790227 \\
\hline $\mathrm{H}$ & 0.328746 & 2.696396 & -3.653294 \\
\hline
\end{tabular}




$\begin{array}{lrrc}\mathrm{H} & 0.592930 & 1.155293 & -2.791277 \\ \mathrm{H} & 3.776208 & 5.699095 & 0.408828 \\ \mathrm{H} & 2.087935 & 6.173803 & 0.737531 \\ \mathrm{H} & 2.818271 & 6.508672 & -0.848570 \\ \mathrm{H} & 2.670270 & 0.099079 & 0.413596 \\ \mathrm{~N} & -1.141707 & 1.020639 & 0.376941 \\ \mathrm{C} & -1.694061 & 1.564240 & -0.716315 \\ \mathrm{C} & -3.063332 & 1.622330 & -0.939970 \\ \mathrm{C} & -3.937736 & 1.094528 & 0.016091 \\ \mathrm{C} & -3.361518 & 0.561863 & 1.173590 \\ \mathrm{C} & -1.977051 & 0.543942 & 1.310915 \\ \mathrm{H} & -0.995646 & 1.949077 & -1.460794 \\ \mathrm{H} & -3.438269 & 2.031374 & -1.878258 \\ \mathrm{C} & -5.403784 & 1.076501 & -0.203768 \\ \mathrm{H} & -3.981434 & 0.164257 & 1.977393 \\ \mathrm{H} & -1.509098 & 0.111373 & 2.197183 \\ \mathrm{C} & -6.043367 & 2.133154 & -0.865305 \\ \mathrm{C} & -7.419378 & 2.108342 & -1.076687 \\ \mathrm{C} & -8.176956 & 1.023955 & -0.635332 \\ \mathrm{C} & -7.550330 & -0.033912 & 0.022142 \\ \mathrm{C} & -6.174919 & -0.006892 & 0.238670 \\ \mathrm{H} & -5.459007 & 2.994390 & -1.193382 \\ \mathrm{H} & -7.903875 & 2.943817 & -1.583259 \\ \mathrm{H} & -9.254383 & 1.004043 & -0.802360 \\ \mathrm{H} & -8.134756 & -0.888251 & 0.365323 \\ \mathrm{H} & -5.687796 & -0.845509 & 0.739447\end{array}$




\section{References}

(1) Schmittel, M.; He, B.; Mal, P. Supramolecular Multicomponent Self-Assembly of Shape-Adaptive Nanoprisms: Wrapping up $\mathrm{C}_{60}$ with Three Porphyrin Units. Org. Lett. 2008, 10, 2513-2516.

(2) Fujita, M.; Oka, H.; Ogura, K. Palladium(0)/LiCl Promoted Cross-Coupling Reaction of (4-Pyridyl)stannanes and Aromatic Bromides: Easy Access to Poly(4-pyridyl)-Substituted Aromatics. Tetrahedron Lett. 1995, 36, 5247-5250.

(3) Hoffmann, M.; Kärnbratt, J.; Chang, M.-H.; Herz, L. M.; Albinsson, B.; Anderson, H. L. Enhanced $\pi$ Conjugation around a Porphyrin[6] Nanoring. Angew. Chem. Int. Ed. 2008, 47, 4993-4996.

(4) Zhu, B.; Chen, H.; Lin, W.; Ye, Y.; Wu, J.; Li, S. Template-Directed Synthesis of Flexible Porphyrin Nanocage and Nanorings via One-Step Olefin Metathesis. J. Am. Chem. Soc. 2014, 136, 15126-15129.

(5) Gaussian 16, Revision A.03, Frisch, M. J.; Trucks, G. W.; Schlegel, H. B.; Scuseria, G. E.; Robb, M. A.; Cheeseman, J. R.; Scalmani, G.; Barone, V.; Petersson, G. A.; Nakatsuji, H.; Li, X.; Caricato, M.; Marenich, A. V.; Bloino, J.; Janesko, B. G.; Gomperts, R.; Mennucci, B.; Hratchian, H. P.; Ortiz, J. V.; Izmaylov, A. F.; Sonnenberg, J. L.; Williams-Young, D.; Ding, F.; Lipparini, F.; Egidi, F.; Goings, J.; Peng, B.; Petrone, A.; Henderson, T.; Ranasinghe, D.; Zakrzewski, V. G.; Gao, J.; Rega, N.; Zheng, G.; Liang, W.; Hada, M.; Ehara, M.; Toyota, K.; Fukuda, R.; Hasegawa, J.; Ishida, M.; Nakajima, T.; Honda,Y.; Kitao, O.; Nakai, H.; Vreven, T.; Throssell, K.; Montgomery, J. A.; Peralta Jr., J. E.; Ogliaro, F.; Bearpark, M. J.; Heyd, J. J.; Brothers, E. N.; Kudin, K. N.; Staroverov, V. N.; Keith, T. A.; Kobayashi, R.; Normand,J.; Raghavachari, K.; Rendell, A. P.; Burant, J. C.; Iyengar, S. S.; Tomasi, J.; Cossi, M.; Millam, J. M.; Klene, M.; Adamo, C.; Cammi, R.; Ochterski, J. W.; Martin, R. L.; Morokuma, K.; Farkas, O.; Foresman J. B.; Fox, D. J. Gaussian, Inc., Wallingford CT, 2016.

(6) Zhao Y.; Truhlar, D. G. A New Local Density Functional for Main-group Thermochemistry, Transition Metal Bonding, Thermochemical Kinetics, and Noncovalent Interactions. J. Chem. Phys. 2006, 125, 194101. 
(7) Schaefer, A.; Huber, C.; Ahlrichs, R. Fully Optimized Contracted Gaussian Basis Sets of Triple Zeta Valence Quality for Atoms Li to Kr. J. Chem. Phys. 1994, $100,5829$.

(8) Dunning Jr., T. H. Gaussian Basis Sets for Use in Correlated Molecular Calculations. I. The Atoms Boron through Neon and Hydrogen. J. Chem. Phys. 1989, 90, 1007.

(9) Blanco, M. A.; Pendás, A. M.; Francisco, E. Interacting Quantum Atoms: A Correlated Energy Decomposition Scheme Based on the Quantum Theory of Atoms in Molecules. J. Chem. Theory Comput. 2005, 1, 1096-1109.

(10) Francisco, E.; Pendás, A. M.; Blanco, M. A. A Molecular Energy Decomposition Scheme for Atoms in Molecules. J. Chem. Theory Comput. 2006, 2, 90-102.

(11) ADF2018, SCM, Theoretical Chemistry, Vrije Universiteit, Amsterdam, The Netherlands, 2018. 